\title{
Preferences over consumption and status
}

\author{
Alexander Vostroknutov
}

Published online: 12 January 2012

C The Author(s) 2012. This article is published with open access at Springerlink.com

\begin{abstract}
Experimental evidence suggests that individual consumption has not only personal value but also enters the social part of the utility. Existing models of social preferences make ad hoc parametric assumptions about the nature of this duality. This creates a problem of experimental identification of preferences since without such assumptions it is impossible to distinguish whether consumption or social concerns are driving the behavior. Given observed choice, the Axiomatic model of preferences in this article makes it possible to unambiguously determine personal and social utility without any assumptions about their relationship. The unique separation can be achieved only if the individual choices in different subgroups of other people are available. Preferences over consumption and status are used as an example to demonstrate how the utility is constructed. The model shows what kind of information about choice is needed to empirically determine the nature of social preferences without making restrictive assumptions. This can help to estimate whether personal consumption or social value is more important in economic decisions.
\end{abstract}

Keywords Axiomatic systems - Experiments - Social preferences - Status · Subjective probability

JEL Classification D01 · D11 · C90

\section{Introduction}

It is a well-established fact that people have strong tendency to compare themselves with their social group. Deviations from selfishness due to distributional concerns were found in many experimental settings: markets (Ball et al. 2001); public goods

\footnotetext{
A. Vostroknutov $(\varangle)$

Department of Economics, Maastricht University, P.O. Box 616, 6200 MD Maastricht, The Netherlands e-mail: a.vostroknutov@maastrichtuniversity.nl
} 
(Andreoni 1995; Fehr and Gächter 2000); Ultimatum games (Güth et al. 1982; CostaGomes and Zauner 2001). One way to rationalize the behavior in these experiments is to assume that utility is a function of the payoffs of others. This modeling technique was used in several studies: inequality aversion, reciprocity, and fairness (Fehr and Schmidt 1999; Bolton and Ockenfels 2000; Rabin 1993); altruism and spitefulness (Levine 1998); status seeking (Frank 1985). The goal of this avenue of research is to find social utility that explains the behavior in as many settings as possible. The following principle is generally used. An assumption is made about the nature of interdependence in preferences, for example, inequality aversion, status seeking, or altruism; some parametric functional form is proposed and the estimates of its parameters are found from the data.

There are several difficulties with this approach. First, the behavior in many experiments can be consistent with different assumptions on the nature of interdependence. For example, proposing non-zero amount in the Dictator game can be explained by inequality aversion, altruism, or even status seeking. ${ }^{1}$ Likewise, the behavior in the Ultimatum game can be explained by any of the three models. Second, it is impossible to pin down relative importance of personal and social utility in the environments where personal and social incentives are aligned. For example, people's preference for big cars over small ones can have very different driving force: it can be simply personal preference; it can be an attempt to "keep up with the Jonses;" or it can be the desire for less inequality (given that your car is smaller than average). Third, the models cited above are normally tested in experiments that involve games, thus confounding the effects of interdependence with intentionality (McCabe et al. 2003) which plays important role in game theoretic settings (Falk et al. 2008). ${ }^{2}$

The problems mentioned above make it hard to conclude what is the true nature of interdependence in preferences and how to determine it unambiguously from the observed choice. There are many reasons why knowing this is important. Sobel (2009) and Dufwenberg et al. (2008) show that separable other-regarding preferences are necessary to achieve market efficiency. Therefore, testing separability hypothesis is crucial for applicability of theories based on general equilibrium. Another important factor is the shape of social utility. Suppose that we discovered that people are more inequality averse than status seeking. In this case, government policies directed at more equal wealth distribution can increase social welfare. However, if the reverse is true, such policy can lead to inefficiency as people might allocate resources away from consumption and toward status competition. If status seeking is prevalent then taxing consumption might be Pareto improving as it will decrease excessive spending on status goods (Layard 1980). This is the case because utility from status does not change if everyone decreases their status goods consumption, but personal utility increases as the money once spent on status goods is now spent on personal consumption goods.

Another empirical question is How big is social utility comparing to the personal one? For example, it is clear that in many environments people spend resources on

\footnotetext{
1 Cummins (2005) discusses the evidence of high-status individuals proposing "gifts" or "protection" to low-status ones without expecting anything in return.

2 Players in the extensive form game show intentionality when they try to understand others' intentions after some choice has been made. For example, McCabe et al. (2003) show that simple addition of an action in the beginning of the game that is rarely taken influences the consecutive behavior of the second movers.
} 
status competitions. If status is relatively unimportant, then policies to reduce overconsumption might not be necessary. If the opposite is true, as argued in classical studies of Smith (1759) and Veblen (1899), then government can increase welfare by eliminating the incentives to be involved in status seeking. ${ }^{3}$ The knowledge of the structure of interdependence in preferences is important in different areas of economic research. For example, it can suggest better policies for designing incentives inside the firm (Auriol and Renault 2008); bring better understanding of international economic migration (Massey et al. 1993); or help explain consumption patterns (Charles et al. 2009).

This article is an attempt to develop a theoretical framework that makes it possible to uncover social preferences from the observed choices. Within this framework, it should be also possible to make absolute quantitative comparisons between social and personal preferences and between preferences revealed in different environments. I show that if preferences are consistent with certain Axioms, then, in the equivalent utility representation, the shape of social utility as well as its size relative to the personal utility can be determined unambiguously. In order to achieve this unique identification, I assume that the choices of the same person are observed in more than one social group. The difference in preferences between the groups is then used to determine the utility.

The search for the Axiomatic systems and equivalent representations in this article is guided by the desire to have simple testable Axioms and tractable utility that can be used in further research. The proposed Axioms are simple and easy to test and the utility form that they imply has straightforward meaning with few parameters. The framework, therefore, suggests experimental designs and points at the requirements for the empirical data that are necessary for testing the hypotheses about the properties of social utility. This makes it possible to find out the nature of interdependence without using parametric assumptions on the shape of the utility and without confounding it with intentionality.

The shape of the utility is limited only by assumptions and Axioms necessary for unique separability of consumption and social utilities (see Definitions 1 and 3 and Axioms E1-E5). ${ }^{4}$ However, I use status seeking to illustrate how the model works. There are several reasons for this. First, the desire for both status and consumption drive the behavior in the same direction, thus making it most challenging to separate their effects. Second, growing body of literature supports the hypothesis that envy and the resulting desire for status are evolved traits of humans beings (Cummins 2005). Economic experiments also confirm this (Vostroknutov et al. 2011).

Ok and Koçkesen (2000) study similar model in which the agent has preferences over her possessions and the distribution of possessions of others (though without considering different social groups). They describe the Axioms that give rise to the negatively interdependent preferences that depend only on the possessions of the agent and the mean of the distribution. The authors overcome the unidentifiability problem by assuming that personal consumption is linear in the amount of goods and interdependence in preferences is negative (status). In my approach, the possibility of

\footnotetext{
${ }^{3}$ See De Graaf et al. (2005) for a variety of anecdotal examples of overconsumption.

${ }^{4}$ In particular, certain forms of altruistic utility cannot be covered in proposed framework (see discussion after Axiom E4.).
} 
comparison between groups makes it possible to have arbitrary interdependence and consumption parts of the utility function.

The article is organized as follows. In Sect. 2, I use examples to discuss conceptual problems with separability of social and personal parts of the preferences. Section 3 describes the basic framework and two approaches to representing the social part of the preferences. In Sects. 4 and 5, the models with single and multiple distributions are described. Section 6 contains discussion on why group size might matter for social preferences. Proofs of the theorems and lemmata can be found in Sects. 7 and 8. Section 8 contains some additional results.

\section{Separability of consumption and status}

People choose to buy some goods purely for consumption purposes, for example cheap food. Other goods are bought for status reasons. However, some goods are chosen for both reasons at once. A good example is cars. People like cars because they are convenient. However, it can hardly be denied that certain cars are produced and bought for status reasons as well.

In order to model social preferences, it is, thus, important to have consumption and social parts of the utility intertwined. How should these parts be represented? The consumption part of the preferences should be independent of anything related to others. Consider a man stranded on an island alone with a luxurious car. We can find out the utility $U_{\mathrm{c}}\left(x_{0}\right)$ that the man derives from the car since it can still be used to take him from point A to B. ${ }^{5}$ This utility is pure consumption utility, independent of anything related to others since there is no one else on the island. Now suppose that suddenly a group of tourists arrive together with their cars. If the man is status seeking, he would compare his car to other cars and prefer his car to be better than as many of them as possible. Thus, the utility of the car might change to, say, $U\left(x_{0}, \mu\right)$ where $\mu$ represents the characteristics of all other cars. However, it is still safe to assume that the man keeps liking his car as a mean of transportation. Therefore, I assume that $U\left(x_{0}, \mu\right)=U_{\mathrm{c}}\left(x_{0}\right)+U_{\mathrm{s}}\left(x_{0}, \mu\right)$ where $U_{\mathrm{s}}\left(x_{0}, \mu\right)$ represents the social utility that "appeared" once others have arrived. ${ }^{6}$

Now, suppose we can only observe the man in the presence of others. We deduce his utility to be $U_{\mathrm{c}}\left(x_{0}\right)+U_{\mathrm{s}}\left(x_{0}, \mu\right)$. But can we claim that $U_{\mathrm{c}}\left(x_{0}\right)$ is the consumption utility and $U_{s}\left(x_{0}, \mu\right)$ is the social utility? Here is a problem. Choose any function $g\left(x_{0}\right)$ and redefine the utility as $U\left(x_{0}, \mu\right)=g\left(x_{0}\right)+\bar{U}_{s}\left(x_{0}, \mu\right)$, where $\bar{U}_{s}\left(x_{0}, \mu\right)=$ $U_{s}\left(x_{0}, \mu\right)+U_{\mathrm{c}}\left(x_{0}\right)-g\left(x_{0}\right)$. So, now $g$ becomes the utility for consumption! This shows that it is impossible in principle to separate status or any other social preference from consumption in a unique way when we observe individual choices that conform with utility $U$.

One way around this is to assume that we can observe the choices of the agent inside different social groups as well as between the groups. Consider another example of

\footnotetext{
$5 x_{0}$ represents some characteristics of the car.

6 Similar additive structure is used in Maccheroni et al. (2008). Bell and Keeney (2008) also use additive utility.
} 
preferences of a game theorist over the number of publications in top economics journals and two social groups: "economists" and "biologists." Assume that, if the game theorist has any social preferences at all, she cares more about economists than biologists. This means that there are distributions of possessions of others such that she is not indifferent to which group to belong (given some fixed number of personal publications). ${ }^{7}$

If game theorist reveals that she prefers to have more publications than everybody else while being among economists and she does not care about the number of her publications while being among biologists (regardless of their publications in top economics journals), then we can conclude that she does not derive any personal utility from the number of publications, and that her true social preferences are revealed through the comparisons with other economists. Such relations of preferences inside different social groups therefore can uncover the shape of social utility.

Another important piece of information about preferences can be elicited from the choices between groups. Imagine that game theorist reveals that she cares about relative number of her publications among economists as well as among biologists but to lesser extent. Also suppose that, when asked to which group she wants to belong, game theorist is indifferent as long as all economists and biologists do not have any publications. This means that for the specific distribution of publications of others (nobody has any) game theorist chooses as if groups do not exist. This information can be used to reconstruct the personal part of her preferences which is the same across two groups. The answers to the questions like "Do you prefer to have $a$ or $b$ publications given that no one else has any?" thus reveal her personal preference for own publications. After that, social preferences in each group can be found by "subtracting" the unique personal preferences. ${ }^{8}$

I show that it is possible to construct utility, unique up to a positive affine transformation, as long as the comparisons like the above can be made. In the example, preferences can be represented by two functions: $U_{1}\left(x_{0}, \mu\right)=U_{\mathrm{c}}\left(x_{0}\right)+U_{s 1}\left(x_{0}, \mu\right)$ and $U_{2}\left(x_{0}, \mu\right)=U_{\mathrm{c}}\left(x_{0}\right)+U_{s 2}\left(x_{0}, \mu\right)$ which correspond to the two groups. Notice that the personal utility $U_{\mathrm{c}}$ is group independent. The pivotal assumptions that are necessary for this result are (1) preferences are observed inside different groups and between them; (2) there are distributions of possessions of others such that the agent cares to which group to belong; (3) there are distributions of possessions of others such that the agent does not care to which group to belong.

\section{The framework}

The world consists of agent 0 and a finite set $S$ of other agents with $|S|>1$ and $\{0\} \notin S$. We are interested in modeling the preferences of agent 0 . Agent 0 has the

\footnotetext{
7 In this example, I assume that the game theorist has intrinsic preferences over the number of publications. Another valid approach is to see the desire to have more publications than others as means to achieving more personal consumption (Postlewaite 1998).

8 If there are more than two groups, then, as long as the game theorist is indifferent between any two groups given the same possessions of others (no one has any publications), the same argument can be used to find social preferences in all groups.
} 
measure of possessions and social status $x_{0} \in X$, where $X$ is any non-empty set with $|X|>2$. This measure can be some aggregate that is calculated using the possessions or some qualities of the agent, depending on the social group of interest. For example, it can be the money value of all the goods that the agent has $\left(X=\mathbb{R}_{+}\right)$, or it can be the possessions themselves $\left(X=\mathbb{R}_{+}^{n}\right) .{ }^{9}$ The crucial assumption is that $x_{0}$ plays dual role of bringing not only consumption but also social benefit.

Think of $S$ as a "big" set of all people that agent 0 can possibly care about. This can be, for example, people of the same profession, like all economists, or any other big social group. It is realistic to assume that at any given time agent 0 does not take into consideration everybody in $S$, but only some subset $T \subseteq S$. This subset can be, for example, the people that are geographically close to agent 0 (economists on the same economics department). An intuition behind this assumption might be like this: everybody in $T$ observes the possessions (social status) of agent 0 , because they are close to him and everybody else in $S \backslash T$ does not directly see agent 0's possessions (status); agent 0 does not take into account the people who do not see his possessions, but can potentially do so if they become aware of them (for example, if agent 0 moves to different city, or another economics department).

The final assumption that I am making is that agent 0 observes the possessions (status) of other agents in $T$. In particular, he sees the distribution of possessions of others in set $X$ and can express preferences over his $x_{0} \in X$ and distributions over statuses of others in a given subgroup. In addition, I assume that agent 0 can report to which of the two groups of others he would like to belong, given proposed levels of his status and the distributions of possessions of others in both groups.

In principle, it is possible to have the utility representation for the preferences which are defined only on elements of $X$ without any uncertainty. However, this approach faces a problem of multiplicity of utility functions (not up to a positive affine transformation). The assumption that preferences are defined over distributions of possessions eliminates this problem making utility representation unique (up to a positive affine transformation).

In the following two sections, I explore two different ways in which agent 0 can see the distributions of others. One possibility is that for any group $T \subseteq S$ agent 0 regards the possessions of each individual in $T$ as coming from a single distribution (Sect. 4). Another possibility is that agent 0 considers separate distribution for each individual agent in $T$ (Sect. 5). The goal for both cases is to find a simple utility representation and the set of simple Axioms equivalent to this representation that would allow for an intuitive way of separating consumption and status preferences.

\section{The model with single distribution}

Assume that there are two fixed subgroups of others $S_{1}$ and $S_{2} \cdot{ }^{10}$ Suppose that agent 0 has information only about distributions of possessions of others in these two groups and no information about particular individuals inside the groups. From the empirical

\footnotetext{
9 The set $X$ can be any set. Analogy with reals is brought only for illustration.

10 The axiomatization below can be easily generalized to any number of groups with minor modifications.
} 
perspective this situation happens, for example, when agent 0 faces a decision to which neighborhood to move in and he has information about income distributions in each of them. From the individual or game theoretic perspective, $S_{1}$ and $S_{2}$ can be thought of as two individuals. In this case, agent 0 considers the distributions of their possessions. Regardless of the interpretation, the important assumption here is that agent 0 thinks of only one distribution of possessions attached to each group.

The preferences of agent 0 are defined over two separate sets $\Delta\left(X^{2}\right)$ of simple lotteries over $X^{2}{ }^{11}$ The two copies of $\Delta\left(X^{2}\right)$ represent agent 0 's possessions and his possible observations of the possessions of others in groups $S_{1}$ and $S_{2}$. For any fixed group and lottery, the first component of $X^{2}$ represents the possessions of agent 0 . The conditional distribution of the second component given fixed $x_{0}$ from the support of the first component represents agent 0 's possible observation of possessions of all other agents in $S_{1}$ or $S_{2}$. For example, the degenerate lottery $\left(x_{0}, x\right) \in \Delta\left(X^{2}\right)$ represents the situation in which agent 0 has possessions $x_{0}$ and each agent in, say, $S_{1}$ has possessions $x$. I assume that agent 0 might not know his own possessions with certainty, but he knows the probabilities of realizations of his possessions and the observations of the possessions of others, given those realizations. Let us assume that we observe agent 0 's preferences over all lotteries $\mathcal{E}_{S_{1}}=\mathcal{E}_{S_{2}}:=\Delta\left(X^{2}\right)$ in both groups of others. Let $\mathcal{E}:=\mathcal{E}_{S_{1}} \cup \mathcal{E}_{S_{2}}$ be the set of all possible lotteries over agent 0 's possessions and possessions of others in both groups and let $\succcurlyeq$ be a preference relation over $\mathcal{E}$ with $\sim$ and $\succ$ being its symmetric and asymmetric parts.

The following notation will be used in this section. Let $\mathcal{S}=\left\{S_{1}, S_{2}\right\}$ be a two element set. For $T \in \mathcal{S}$ write $\left(x_{0}, \eta\right)_{T} \in \mathcal{E}_{T}$ as an element of $\Delta\left(X^{2}\right)$ such that the possessions of agent 0 are fixed at $x_{0}$ and the possessions of all other agents in $T$ are distributed according to $\eta \in \Delta(X)$. The expectation of a function with two arguments is written as $E_{h}\left[v\left(x_{0}, x\right)\right]$ for $h \in \Delta\left(X^{2}\right)$, or as $E_{\eta}\left[v\left(x_{0}, \cdot\right)\right]$ for $\eta \in \Delta(X)$, with first argument fixed.

\subsection{Separation of consumption and status}

In this section, the minimal number of Axioms is given that allows for the intuitive separation of consumption from status. The first set of Axioms are the standard conditions necessary to obtain expected utility representation for the preferences.

$\mathbf{E 1} \succcurlyeq$ is reflexive, transitive, total, ${ }^{12}$ and non-trivial:

1.1 For all $T \in \mathcal{S}$, there are $x_{0} \in X$ and $\eta, v \in \Delta(X)$ such that $\left(x_{0}, \eta\right)_{T} \succ$ $\left(x_{0}, v\right)_{T}$

1.2 There are $x_{0}^{*} \in X$ and $\eta^{*} \in \Delta(X)$ such that $\left(x_{0}^{*}, \eta^{*}\right)_{S_{1}} \succ\left(x_{0}^{*}, \eta^{*}\right)_{S_{2}}$

E2 Independence. For all $T \in \mathcal{S}$, all $h, z, w \in \mathcal{E}_{T}$ and all $\alpha \in(0,1)$

$$
h \succ z \Longrightarrow \alpha h+(1-\alpha) w \succ \alpha z+(1-\alpha) w
$$

\footnotetext{
11 Set $\Delta\left(X^{2}\right)$ consists of all distributions $\left(\left(x_{0 i}, x_{i}\right) \circ p_{i}\right)_{i=1 \ldots N}$ with finite support. Here $x_{0 i}$ is the possessions of agent $0, x_{i}$-possessions of others in one of the two groups, and $p_{i}$-the probability of the occurrence of the pair $\left(x_{0 i}, x_{i}\right)$.

12 Totality: $a \neq b \Rightarrow[a \succcurlyeq b \vee b \succcurlyeq a]$.
} 
E3 Continuity. For all $T \in \mathcal{S}$, all $h, z, w \in \mathcal{E}_{T}$ there exist $\alpha, \beta \in(0,1)$

$$
h \succ z \succ w \Longrightarrow \alpha h+(1-\alpha) w \succ z \succ \beta h+(1-\beta) w
$$

Apart from the standard weak order assumptions, Axioms $E 1$ requires that $\succcurlyeq$ is nontrivial in two different ways: E1.1 asks for agent 0 to care about what others have; E1.2 asks for the existence of a pair $\left(x_{0}^{*}, \eta^{*}\right)$ such that agent 0 , given the choice between two groups, prefers being in group $S_{1}$ rather than $S_{2}$ with the same level of his and others' possessions. Throughout the exposition, the asymmetry between $S_{1}$ and $S_{2}$ will be maintained: agent 0 will be assumed to care more about $S_{1}$.

Axioms E2 and E3 are standard requirements for $\succcurlyeq$ to have expected utility representation. Notice that these Axioms restrict $\succcurlyeq$ only inside each fixed group and they say nothing about the choices between groups. When eliciting preferences from choices between lotteries, it might happen that E2 and/or E3 are violated (e.g., common ratio effect). However, the meta-analysis of many decision making experiments (see Starmer (2000) for overview) shows that EU violations become salient only for choices close to the border of the probability simplex. Thus, eliciting EU preferences with lotteries in the middle of the simplex should be possible.

The next two Axioms are the minimal conditions that allow for the intuitively sensible way to disentangle consumption part of the preferences from the status part (or many other types of interdependency). ${ }^{13}$ The main idea is to fix agent 0 's possessions $x_{0} \in X$ and the possessions of others $\eta \in \Delta(X)$ and see how he chooses between the two groups. There are two possibilities: (1) agent 0 prefers one group to the other: $\left(x_{0}, \eta\right)_{S_{1}} \succ\left(x_{0}, \eta\right)_{S_{2}} ;(2)$ agent 0 is indifferent: $\left(x_{0}, \eta\right)_{S_{1}} \sim\left(x_{0}, \eta\right)_{S_{2}}$. In the former case, since everything but the group identity is fixed, agent 0 's choice must have been influenced only by the properties of the groups. In the latter case, there are two possible explanations: agent 0 is indifferent, because he does not care at all to which group to belong (which is ruled out by E1.2); or he does care about the groups, but is indifferent in this particular case because of some property of $\eta$ (for example, as long as everybody has the same amount as agent 0 , he does not care which group to be with). This last possibility gives us the way to reveal the consumption part of agent 0 's preferences. Suppose that we somehow established that agent 0 is indifferent between $\left(x_{0}, \eta\right)_{S_{1}}$ and $\left(x_{0}, \eta\right)_{S_{2}}$ because of some property of $\eta$ and that the same holds for some $\left(y_{0}, v\right)_{S_{1}}$ and $\left(y_{0}, v\right)_{S_{2}}$. For these two pairs, the effects of differences between groups are offset by the special properties of $\eta$ and $\nu$. Therefore, agent 0 's preferences over $\left(x_{0}, \eta\right)_{S_{1}}$ and $\left(y_{0}, v\right)_{S_{1}}$ are influenced only by the consumption benefits of $x_{0}$ and $y_{0}$. The following Axioms make sure that comparisons like this can be made.

\section{E4 Group Indifference.}

4.1 For all $x_{0} \in X$, there is $x^{*} \in X$ such that $\left(x_{0}, x^{*}\right)_{S_{1}} \sim\left(x_{0}, x^{*}\right)_{S_{2}}$

4.2 For any $\ell, m \in \Delta\left(X^{2}\right)$ and $\alpha \in[0,1]$, it is true that

$$
\left[\ell_{S_{1}} \sim \ell_{S_{2}} \wedge m_{S_{1}} \sim m_{S_{2}}\right] \Longrightarrow(\alpha \ell+(1-\alpha) m)_{S_{1}} \sim(\alpha \ell+(1-\alpha) m)_{S_{2}}
$$

13 Certain types of social preferences cannot be represented in this framework. See explanations after Axiom E4. 
Axiom E4.1 requires that for any $x_{0}$, we can find some level of possessions of others $x^{*} \in X$ such that agent 0 is indifferent between the groups. If such element does not exist for some $x_{0}$, then we cannot use the procedure described above to separate the consumption effect. Indeed, suppose that for any $\eta \in \Delta(X)$ we have strong preference between $\left(x_{0}, \eta\right)_{S_{1}}$ and $\left(x_{0}, \eta\right)_{S_{2}}$. Assume that $\succcurlyeq$ can be represented by some utility function. Then, the utilities of $\left(x_{0}, \eta\right)_{S_{1}}$ and $\left(x_{0}, \eta\right)_{S_{2}}$ have to depend on some properties of the groups all the time, thus making it impossible to disentangle the consumption part (see Sect. 4.2 for the additional discussion). This creates certain restrictions on the types of social preferences that can be studied in this framework. For example, if $S_{1}$ is an in-group and $S_{2}$ is an out-group and agent 0 has altruistic preferences for the in-group but not for the out-group, then he will always have preference $\left(x_{0}, \eta\right)_{S_{1}} \succ\left(x_{0}, \eta\right)_{S_{2}}$ for all $x_{0}$ and $\eta$. Here, in-group has special meaning to agent 0 which makes it impossible to fit into this framework.

E4.2 asks for some consistency when the mixtures of indifferent pairs are considered. Given two indifferent pairs, the same mixture of them in both groups keeps agent 0 indifferent.

E5 Group Disparity. For all $x_{0} \in X$ and $\eta, v \in \Delta(X)$ with $\left(x_{0}, \eta\right)_{S_{1}} \sim\left(x_{0}, \eta\right)_{S_{2}}$

$$
\begin{aligned}
& \left(x_{0}, v\right)_{S_{2}} \succ\left(x_{0}, \eta\right)_{S_{2}} \Longrightarrow\left(x_{0}, v\right)_{S_{1}} \succ\left(x_{0}, v\right)_{S_{2}} \text { and } \\
& \left(x_{0}, \eta\right)_{S_{2}} \succ\left(x_{0}, v\right)_{S_{2}} \Longrightarrow\left(x_{0}, v\right)_{S_{2}} \succ\left(x_{0}, v\right)_{S_{1}}
\end{aligned}
$$

Axiom E5 establishes the way in which agent 0 can care differently about the two groups. It says that agent 0 always cares more about $S_{1}$ than about $S_{2}$ : for any indifferent pair $\left(x_{0}, \eta\right)_{S_{1}} \sim\left(x_{0}, \eta\right)_{S_{2}}$, if agent 0 prefers some $\left(x_{0}, v\right)$ in $S_{2}$ to $\left(x_{0}, \eta\right)$ in $S_{2}$ then he should like it even more in $S_{1}$ and vice versa. This Axiom (and E5 ${ }^{\star}$ below) assumes asymmetry between $S_{1}$ and $S_{2}$. The Axiom could have also asked for one of the two groups to play the role of $S_{1}$. This can be done by switching the names of the groups instead.

To illustrate the intuition think of the groups of economists and biologists, an economist would value his academic success higher if other economists know about it rather than when biologists know about it and other way round: he would be more unhappy if other economists get aware of his academic misfortune than when biologists find out about it. This Axiom essentially establishes the desired property of the indifference pairs $\left(x_{0}, \eta\right)_{S_{1}} \sim\left(x_{0}, \eta\right)_{S_{2}}$ : the indifference between the groups can result only from the special properties of $\eta$ which are group independent (see Sect. 4.2 for the additional discussion).

Axioms E1-E5 are enough for the desired utility representation (see Theorem 2). However, the result comes with the conditions on the utility functions that are hard to verify (conditions (4.3) and (4.4)). Therefore, I first present a little bit less general result which requires an additional Axiom. In this representation, the utility functions are well behaved.

E6 Likes and Dislikes. For any $T \in \mathcal{S}, x_{0} \in X$ and $\eta \in \Delta(X)$ with $\left(x_{0}, \eta\right)_{S_{1}} \sim$ $\left(x_{0}, \eta\right)_{S_{2}}$

$$
\begin{array}{ll}
\text { either } \forall v \in \Delta(X) & \left(x_{0}, v\right)_{T} \sim\left(x_{0}, \eta\right)_{T} \\
\text { or } \exists v, \tilde{v} \in \Delta(X) & \left(x_{0}, v\right)_{T} \succ\left(x_{0}, \eta\right)_{T} \succ\left(x_{0}, \tilde{v}\right)_{T}
\end{array}
$$


The Axiom requires that for each $x_{0} \in X$ only one of the two situations is possible: (1) agent 0 is indifferent between any possessions of others, given $x_{0}$ or (2) there are possessions of others that make agent 0 both happier and unhappier in both $S_{1}$ and $S_{2}$ than his consumption level. Essentially, this rules out two unlikely cases when agent 0 just likes others to be around or hates others to be around (again, in comparison to his consumption level, that is represented by the indifference pair). The Axiom is not particularly demanding, but, amazingly, it allows for much nicer utility function. ${ }^{14}$

The following Definitions and Theorem give the representation:

Definition 1 Call any $v: X^{2} \rightarrow \mathbb{R}$ a status function if it is not constant and there is an $x^{*}\left(x_{0}\right) \in X$ such that $v\left(x_{0}, x^{*}\left(x_{0}\right)\right)=0$ for all $x_{0} \in X$.

Status functions will play the role of the social part of the preferences representation. The condition that for all $x_{0}$, there is $x^{*}\left(x_{0}\right)$ such that $v\left(x_{0}, x^{*}\left(x_{0}\right)\right)=0$ might look restrictive. However, it is just the reflection of Axiom E4.1 that asks for the existence of $x^{*}$ for each $x_{0}$ such that $\left(x_{0}, x^{*}\right)_{S_{1}} \sim\left(x_{0}, x^{*}\right)_{S_{2}}$. In what follows, status function $v\left(x_{0}, x^{*}\right)$ will be zero if $\left(x_{0}, x^{*}\right)_{S_{1}} \sim\left(x_{0}, x^{*}\right)_{S_{2}}$ (thus only personal part of preferences matters).

Definition 2 Let $g: X \rightarrow \mathbb{R}$ be any function, $v: X^{2} \rightarrow \mathbb{R}$ be status function such that for all $x_{0} \in X$ either $v\left(x_{0}, \cdot\right) \equiv 0$ or there are $x, x^{\prime} \in X$ with $v\left(x_{0}, x\right) / v\left(x_{0}, x^{\prime}\right)<0$, and let $\pi: X \rightarrow \mathbb{R}$ satisfy $\pi\left(x_{0}\right) \in(0,1)$ for all $x_{0}$. For $T \in \mathcal{S}$ let $V_{T}: \mathcal{E}_{T} \rightarrow \mathbb{R}$ be defined as

$$
\begin{aligned}
& V_{S_{1}}[h]=E_{h}\left[g\left(x_{0}\right)+v\left(x_{0}, x\right)\right] \\
& V_{S_{2}}[h]=E_{h}\left[g\left(x_{0}\right)+\pi\left(x_{0}\right) v\left(x_{0}, x\right)\right]
\end{aligned}
$$

Finally, let $V: \mathcal{E} \rightarrow \mathbb{R}$ be equal to $V_{T}[h]$ for all $T \in \mathcal{S}$ and $h \in \mathcal{E}_{T} \cdot{ }^{15}$

\section{Theorem 1 The following two statements are equivalent:}

1. $\succcurlyeq$ satisfies E1-E6

2. $\succcurlyeq$ has a utility representation $V: \mathcal{E} \rightarrow \mathbb{R}$ as described in Definition 2, unique up to a positive affine transformation.

\section{Proof See Sect. 7.}

In the utility representation $g\left(x_{0}\right)+v\left(x_{0}, x\right)$, the first term is the consumption part of the utility and the second term represents the social utility. Notice that, as desired, $g\left(x_{0}\right)$ is common in both utilities. The functions $v\left(x_{0}, x\right)$ and $\pi\left(x_{0}\right) v\left(x_{0}, x\right)$ satisfy two properties: (1) they are equal to zero only when agent 0 does not care about the two groups; (2) whenever they are not zero (there are some group effects) they are different. This reflects the ideas about the separation of consumption described above. In particular, it is never the case that $v\left(x_{0}, x\right)$ and $\pi\left(x_{0}\right) v\left(x_{0}, x\right)$ are equal and not zero. This case is not desirable because it is not clear then whether it is consumption

\footnotetext{
14 In particular, E6 does not put any restrictions on the size of $X$ (see Appendix 8).

15 Notation $E_{h}\left[g\left(x_{0}\right)+v\left(x_{0}, x\right)\right]$ means the expectation of $g+v$ w.r.t. $h \in \Delta\left(X^{2}\right)$. $x_{0}$ and $x$ show where the first and the second components of support of $h$ go.
} 
or social utility that drives agent 0 's preference. Also, in this representation, there is essentially only one social utility function $v\left(x_{0}, x\right)$. For the group $S_{2}$, there is also a weighting function $\pi: X \rightarrow \mathbb{R}$ that tells how much less does agent 0 care about $S_{2}$ than $S_{1}$ for each $x_{0}$.

This representation is compatible with utilities that have reference points. For example, preferences given by utilities $V_{S_{1}}\left(x_{0}, \eta\right)=g\left(x_{0}\right)+\left(x_{0}-E_{\eta}\right)$ and $V_{S_{2}}\left(x_{0}, \eta\right)=$ $g\left(x_{0}\right)+\beta\left(x_{0}-E_{\eta}\right)$ with $\beta \in(0,1)$ satisfy all the Axioms E1-E6. Here, consumption part of the preferences is $g\left(x_{0}\right)$ and social part is given by the difference between $x_{0}$ and the mean of the distribution of the possessions of others $E_{\eta}$.

Now we can move on to the representation result without E6.

Definition 3 Let $g: X \rightarrow \mathbb{R}$ be any function, and $v_{S_{1}}, v_{S_{2}}: X^{2} \rightarrow \mathbb{R}$ be status functions such that for any $x_{0}, x \in X$ and any $\eta \in \Delta(X)$

$$
\begin{aligned}
v_{S_{1}}\left(x_{0}, x\right)=v_{S_{2}}\left(x_{0}, x\right) & \Longleftrightarrow v_{S_{1}}\left(x_{0}, x\right)=0 \\
E_{\eta}\left[v_{S_{1}}\left(x_{0}, \cdot\right)\right] \neq 0 & \Longleftarrow E_{\eta}\left[v_{S_{2}}\left(x_{0}, \cdot\right)\right] \neq 0 \\
\left|E_{\eta}\left[v_{S_{2}}\left(x_{0}, \cdot\right)\right]\right|<\left|E_{\eta}\left[v_{S_{1}}\left(x_{0}, \cdot\right)\right]\right| & \Longleftarrow E_{\eta}\left[v_{S_{2}}\left(x_{0}, \cdot\right)\right] \neq 0
\end{aligned}
$$

For $T \in \mathcal{S}$, let $V_{T}: \mathcal{E}_{T} \rightarrow \mathbb{R}$ be defined as

$$
V_{T}[h]=E_{h}\left[g\left(x_{0}\right)+v_{T}\left(x_{0}, x\right)\right] .
$$

Finally, let $V: \mathcal{E} \rightarrow \mathbb{R}$ be equal to $V_{T}[h]$ for all $T \in \mathcal{S}$ and $h \in \mathcal{E}_{T} \cdot{ }^{16}$

Theorem 2 The following two statements are equivalent:

1. $\succcurlyeq$ satisfies E1-E5

2. $\succcurlyeq$ has a utility representation $V: \mathcal{E} \rightarrow \mathbb{R}$ as described in Definition 3 , unique up to a positive affine transformation.

Proof See Sect. 7.

As can be easily noticed, Theorem 2 allows for more variation in utilities than Theorem 1. In particular, for fixed $x_{0}$, the utilities in two groups don't have to be multiples of one another. However, the drawback is the conditions (4.3) and (4.4). It is not exactly obvious which functions satisfy them and which do not.

Certainty analogs of Axioms E5 and E6 can be used to show the same result in case when preferences are defined only over possessions in $X^{2}$. This, however, is only possible if there is some additional structure on $X$ and $\succcurlyeq$ that would allow for unique utility function (up to a positive affine transformation). It seems more attractive to assume that preferences are defined over distributions than to add ad hoc structure to $X$.

$\overline{16}$ Notation $E_{\eta}\left[v_{S_{1}}\left(x_{0}, \cdot\right)\right]$ means the expectation of $v_{S_{1}}\left(x_{0}, \cdot\right)$ w.r.t. $\eta \in \Delta(X)$. 


\subsection{The role of inter-group Axioms}

I would like to discuss the relevance of inter-group Axioms E4 and E5 to the result of Theorems 1 and 2. Both Axioms are necessary to uniquely disentangle personal and social part of the utility. However, E5 also has some desirable normative properties.

Let us start with E4. Suppose E4 does not hold, then it is true that there exists $x_{0} \in X$ such that for all $x^{*} \in X$ either $\left(x_{0}, x^{*}\right)_{S_{1}} \succ\left(x_{0}, x^{*}\right)_{S_{2}}$ or $\left(x_{0}, x^{*}\right)_{S_{2}} \succ\left(x_{0}, x^{*}\right)_{S_{1}}$. Notice that this strict preference has to come from some difference between the groups as personal possessions $x_{0}$ and possessions of others $x^{*}$ are the same on the left and on the right. Therefore, for $x_{0}$ and any possessions of others $x^{*}$ agent 0 is always not indifferent between the two groups. But then, we cannot obtain a unique separability of utility like in Theorem 2 simply because there is no reference point to determine personal utility $g\left(x_{0}\right)$ anymore. We can choose $g\left(x_{0}\right)$ in any way we want and think of the rest of the utility as social part (the same idea was used in Sect. 2). Axiom E4 is used in the proof of Theorem 2 exactly to uniquely uncover the function $g\left(x_{0}\right)$. This is done in the following way. Suppose E4 holds, then for all $x_{0}$ there is $x^{*}$ with $\left(x_{0}, x^{*}\right)_{S_{1}} \sim\left(x_{0}, x^{*}\right)_{S_{2}}$. The indifference in this case means that agent 0 is indifferent between the two groups given some possessions of others $x^{*}$. In other words, whatever group effects there are they are nullified by the special properties of $x^{*}$ (for example, agent 0 might not care about which group of others to belong to as long as $x_{0}=x^{*}$ ). Now, let us look at the set

$$
X^{*}=\left\{\left(x_{0}, x^{*}\right) \in X^{2}:\left(x_{0}, x^{*}\right)_{S_{1}} \sim\left(x_{0}, x^{*}\right)_{S_{2}}\right\} .
$$

This is the collection of all pairs of possessions that satisfy E4. The set $X^{*}$ has one important property: for any pair in $X^{*}$, agent 0 is indifferent between the groups. Therefore, we can treat the restriction of $\succcurlyeq$ to $X^{*}$ as a genuine personal utility of agent 0 . The fact that by $\mathrm{E} 4 X^{*}$ contains pairs for any $x_{0}$ allows us to completely determine personal utility $g\left(x_{0}\right)$. This would be impossible if some $x_{0}$ were missing, or if E4 did not hold.

Now let us turn to E5. Intuitively, E5 says that agent 0 cares about group $S_{1}$ more than about group $S_{2}$. This is expressed relatively to the pairs of possessions for which agent 0 is indifferent between the groups (elements of $X^{*}$ ). E5 says that given fixed $x_{0}$ if in group $S_{2}$ agent 0 prefers $\left(x_{0}, y^{*}\right)$ to $\left(x_{0}, x^{*}\right)$ then he should like $\left(x_{0}, y^{*}\right)$ in group $S_{1}$ even more. This condition is normative. However, it sounds appealing because it seems unrealistic to think that agent 0 can switch his overall group preference depending on $x_{0}$. The example can be again "all economists" and "all biologists." Economist would care more about academic success (misfortune) among economists than among biologists.

Despite its normative nature Axiom, E5 is also necessary for the unique separation result of Theorem 2. In particular, it rules out the following undesirable case:

$$
\begin{gathered}
\left(x_{0}, x^{*}\right)_{S_{1}} \sim\left(x_{0}, x^{*}\right)_{S_{2}} \\
\curlywedge \\
\left(x_{0}, y^{*}\right)_{S_{1}} \sim\left(x_{0}, y^{*}\right)_{S_{2}}
\end{gathered}
$$

Here $x_{0}$ is fixed, but there are two different possessions of others $x^{*}$ and $y^{*}$ for which agent 0 is indifferent between the two groups. Also, in both groups agent 0 prefers 
$\left(x_{0}, y^{*}\right)$ to $\left(x_{0}, x^{*}\right)$. This situation is undesirable since it is not clear whether $x^{*}$ or $y^{*}$ should be used in the construction of the personal utility as described above. E5 ensures that for any $x^{*}$ and $y^{*}$ if $\left(x_{0}, x^{*}\right)_{S_{1}} \sim\left(x_{0}, x^{*}\right)_{S_{2}}$ and $\left(x_{0}, y^{*}\right)_{S_{1}} \sim\left(x_{0}, y^{*}\right)_{S_{2}}$ then the only situation possible is complete indifference in the diagram above:

$$
\begin{gathered}
\left(x_{0}, x^{*}\right)_{S_{1}} \sim\left(x_{0}, x^{*}\right)_{S_{2}} \\
2 \\
2 \\
\left(x_{0}, y^{*}\right)_{S_{1}} \sim\left(x_{0}, y^{*}\right)_{S_{2}}
\end{gathered}
$$

Thus, the determination of personal utility is unambiguous.

In Appendix 8, it is shown that it is possible to replace E5 with the indifference requirement above (Axiom $5^{\bullet}$ ) and to prove the theorem analogous to Theorem 2 (Theorem 5). In Theorem 5, the conditions (4.3) and (4.4) of Definition 3 are not present anymore. Thus, it is a more general result in which $v_{S_{1}}$ and $v_{S_{2}}$ are only restricted by Definition 1 . However, this generality does not allow for rather appealing utility representations given in Theorems 1 and 3.

\subsection{Linear group disparity}

This section is an attempt to restrict the number of parameters in utility function even further (comparing to Theorem 1). This is done by dropping E6 and replacing E5 with something stronger.

E5 $^{\star}$ Linear Group Disparity. There is $\pi \in(0,1)$ such that for all $x_{0} \in X$ and $\eta, v \in \Delta(X)$

$$
\left(x_{0}, \eta\right)_{S_{1}} \sim\left(x_{0}, \eta\right)_{S_{2}} \Longrightarrow\left(\pi\left(x_{0}, v\right)+(1-\pi)\left(x_{0}, \eta\right)\right)_{S_{1}} \sim\left(x_{0}, v\right)_{S_{2}}
$$

$E 5^{\star}$ requires that there is some fixed probability $\pi$ such that agent 0 is indifferent between having any pair of possessions in $S_{2}$ and having the same pair in $S_{1}$ with probability $\pi$. In terms of the weighting of the social part of the utility, this means that the weight is always the same. Whether this assumption is reasonable or not can be determined experimentally. The following Definition and Theorem give the representation for this case.

Definition 4 Let $g: X \rightarrow \mathbb{R}$ be any function, $v: X^{2} \rightarrow \mathbb{R}$ be status function, and let $\pi \in(0,1)$. For $T \in \mathcal{S}$ let $V_{T}: \mathcal{E}_{T} \rightarrow \mathbb{R}$ be defined as

$$
\begin{aligned}
& V_{S_{1}}[h]=E_{h}\left[g\left(x_{0}\right)+v\left(x_{0}, x\right)\right] \\
& V_{S_{2}}[h]=E_{h}\left[g\left(x_{0}\right)+\pi v\left(x_{0}, x\right)\right]
\end{aligned}
$$

Finally, let $V: \mathcal{E} \rightarrow \mathbb{R}$ be equal to $V_{T}[h]$ for all $T \in \mathcal{S}$ and $h \in \mathcal{E}_{T}$.

Theorem 3 The following two statements are equivalent:

1. $\succcurlyeq$ satisfies E1-E4, E5 * 
2. $\succcurlyeq$ has a utility representation $V: \mathcal{E} \rightarrow \mathbb{R}$ as described in Definition 4, unique up to a positive affine transformation.

Proof See Sect. 7.

\section{The model with multiple distributions}

In the previous section, it was assumed that the agent cares about two groups as separate entities. In particular, he does not care how many people are in each group and what are their personal characteristics. However, there are cases in which group size and individualities of others can matter (see discussion in Sect. 6.1). Therefore, in this section, the model is extended to incorporate these possibilities.

Assume that agent 0 considers separate distribution of possessions for each individual in each group of others. The important difference from the previous section comes from the possibility for other agents to belong to several subgroups simultaneously. I use Anscombe and Aumann (1963) framework to construct preferences that are represented by unique ${ }^{17}$ expected utility function that is given by

$$
U\left(x_{0}, x_{1}, \ldots, x_{T}\right)=f\left(x_{0}\right)+\sum_{i \in T} \pi_{i} u\left(x_{0}, x_{i}\right)
$$

when restricted to the degenerate distributions over $\left(x_{i}\right)_{i \in T}$. Here $x_{0}$ is a measure of possessions of agent 0 , whose preferences are studied. $\left(x_{i}\right)_{i \in T}$ are the same measures for other agents in subgroup $T$ of some set $S$ of all possible others. Agent 0 cares about two things. First, $x_{0}$ has some consumption value. Second, agent 0 derives social value from $x_{0}$ by comparing it to what others have. The consumption part of the utility is represented by $f\left(x_{0}\right)$, whereas the status part is the weighted sum over others. The function $u\left(x_{0}, x\right)$ describes the specific way agent 0 cares about his position relative to one other person and $\left(\pi_{i}\right)_{i \in T}$ are the weights that represent the importance or "closeness" of each other individual to agent 0.

Notice that the desired utility form $U$ in this case, when restricted to the single distribution case (all others have the same distribution over possessions), corresponds to the result of Theorem 3, which was obtained with very strong Axiom E5 ${ }^{\star}$. Below I show that it is possible to obtain representation $U$ without anything like $5^{\star}$.

In order to obtain uniqueness of this representation, it is necessary that the preferences of agent 0 are observed in different subgroups of others. Moreover, in order to obtain unique weights $\left(\pi_{i}\right)_{i \in S}$ for each agent in $S$ and unique function $u$, it is necessary that the observed subgroups have certain degree of intersection. In case of disjoint subgroups, it is impossible to pin down unique function $u$.

The following definition puts the constraints on the observable subgroups.

Definition 5 Say that the collection of observed subgroups $\mathcal{C} \subseteq 2^{S}$ is connected if $\{\emptyset\} \notin \mathcal{C},|\mathcal{C}|>1, \cup \mathcal{C}=S$, and for all $T, R \in \mathcal{C}$ there exist $\bar{C}_{1}, \ldots, C_{K} \in \mathcal{C}$ such that

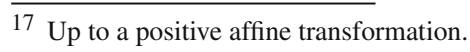




$$
T \cap C_{1} \neq \emptyset, \quad C_{k} \cap C_{k+1} \neq \emptyset, \quad C_{K} \cap R \neq \emptyset \quad \text { where } k=1 \ldots K-1 .
$$

The first three requirements say that (1) we do not observe the behavior of agent 0 in complete solitude (the presence of observer himself makes it impossible); (2) there is more than one subgroup (for otherwise we cannot uniquely separate status and consumption); (3) subgroups cover all other agents (if not, then remove unobserved agents from $S$ ) and (4) any two subgroups can be "connected" by the sequence of intersecting subgroups (otherwise we would have "disconnected" collections of subgroups again making unique identification impossible, see Sect. 2).

Next, let us define for each subgroup $T \subseteq S$ the set of outcomes about which agent 0 cares, or has preferences. It is natural to consider

$$
\chi_{T}:=\Delta\left(X^{\{0\} \cup T}\right)
$$

the set of all simple lotteries over the statuses of agent 0 and all other agents in subgroup $T$.

Choose any connected collection $\mathcal{C} \subseteq 2^{S}$ of subgroups (see Definition 5) and let

$$
\mathcal{A}:=\bigcup_{T \in \mathcal{C}} x_{T}
$$

be the set of all lotteries in $X_{T}$ in all available subsets of other agents. Consider preference relation $\succcurlyeq$ over $\mathcal{A}$ with $\dot{\sim}$ and $\dot{\succ}$ being its symmetric and asymmetric parts.

For $T \in \mathcal{C}, x_{0} \in X$, and $h \in \Delta\left(X^{T}\right)$, write $\left(x_{0}, h_{R}, h_{-R}\right)_{T}$ to emphasize the lotteries corresponding to agents in $R \subseteq T$. For $x_{0}, x \in X$, write $\left(x_{0}, x\right)_{T}$ for the lottery that assigns possessions $x_{0}$ to agent 0 and possessions $x$ to each other agent in $T$.

Define a mixture of two lotteries $h, z \in X_{T}$ with the same domain $T$ to be standard lottery mixture. This turns $\mathcal{X}_{T}$ into a mixture set as defined in Herstein and Milnor (1953). ${ }^{18}$

The following notation is used in the description of the Axioms below. For $h \in X_{T}$ or $p \in \Delta\left(X^{T}\right), \mu_{i}(h)$ (or $\left.\mu_{i}(p)\right)$ stands for the $i$ th marginal distribution. $\Pi \mu_{i}(p) \in$ $\Delta\left(X^{T}\right)$ is the distribution obtained from $p \in \Delta\left(X^{T}\right)$ by taking all marginals and treating them as independent.

Suppose that the following Axioms hold:

A0 Self-Comparison. For all $T \in \mathcal{C}$, all $x_{0} \in X$ and all $p \in \Delta\left(X^{T}\right)$

$$
\left(x_{0}, p\right)_{T} \dot{\sim}\left(x_{0}, \Pi \mu_{i}(p)\right)_{T}
$$

A1 $\succcurlyeq$ is reflexive, transitive, total, and non-trivial: for any $T \in \mathcal{C}$, there are $x_{0}, x, x^{\prime} \in X$ such that

$$
\left(x_{0}, x\right)_{T} \succ\left(x_{0}, x^{\prime}\right)_{T}
$$

\footnotetext{
18 Notice that to obtain the results in this section, it is only necessary for each separate $\chi_{T}$ to be a mixture set. The set $\mathcal{A}$ does not have to possess this property.
} 
A2 Independence. For all $T \in \mathcal{C}$, all $h, z, w \in X_{T}$ and all $\alpha \in(0,1)$

$$
h \succ z \Rightarrow \alpha h+(1-\alpha) w \dot{\succ} \alpha z+(1-\alpha) w
$$

A3 Continuity. For all $T \in \mathcal{C}$, all $h, z, w \in X_{T}$ there exist $\alpha, \beta \in(0,1)$

$$
h \dot{\succ} z \dot{\succ} w \Rightarrow \alpha h+(1-\alpha) w \dot{\succ} z \dot{\succ} \beta h+(1-\beta) w
$$

A4 Anonymity. For all $T \in \mathcal{C}, \mathbf{x} \in X^{T}, i, j \in T$, and $\ell, m \in \Delta\left(X^{2}\right)$

$$
\left(\ell, \mathbf{x}_{-i}\right)_{T} \succcurlyeq\left(m, \mathbf{x}_{-i}\right)_{T} \Longleftrightarrow\left(\ell, \mathbf{x}_{-j}\right)_{T} \succcurlyeq\left(m, \mathbf{x}_{-j}\right)_{T}
$$

A5 Unimportance. For all $x_{0} \in X$ there exists $x^{*}\left(x_{0}\right) \in X$ such that for all intersecting $T, R \in \mathcal{C}$, all $Q \subseteq T \cap R$ and all $x \in X$

$$
\left(x_{0},\left(x^{*}\left(x_{0}\right)\right)_{T \backslash Q},(x)_{Q}\right) \dot{\sim}\left(x_{0},\left(x^{*}\left(x_{0}\right)\right)_{R \backslash Q},(x)_{Q}\right)
$$

A6 Group Disparity. There exist $S_{1}, S_{2} \in \mathcal{C}$ such that for all $x_{0}, x, x^{\prime} \in X$ with $\left(x_{0}, x\right)_{S_{1}} \dot{\sim}\left(x_{0}, x\right)_{S_{2}}$

$$
\begin{aligned}
& \left(x_{0}, x^{\prime}\right)_{S_{1}} \dot{\succ}\left(x_{0}, x\right)_{S_{1}} \Rightarrow\left(x_{0}, x^{\prime}\right)_{S_{1}} \dot{\succ}\left(x_{0}, x^{\prime}\right)_{S_{2}} \text { and } \\
& \left(x_{0}, x\right)_{S_{1}} \dot{\succ}\left(x_{0}, x^{\prime}\right)_{S_{1}} \Rightarrow\left(x_{0}, x^{\prime}\right)_{S_{2}} \dot{\succ}\left(x_{0}, x^{\prime}\right)_{S_{1}}
\end{aligned}
$$

Axioms A1-A3 are standard necessary conditions for existence of an expected utility representation for each $T \in \mathcal{C}$.

Axiom A4 says that agent 0 does not care about the names of the other agents. Given any fixed outcomes for all agents but $i$, if agent 0 prefers lottery $\ell$ to $m$ then he will also prefer $\ell$ to $m$ in a situation when he faces agent $j$ instead of $i$ with all other outcomes still being fixed. Together with the Axioms above, A4 implies that in each restriction $\succcurlyeq_{T}$ agent 0 treats all other agents in $T$ in the same way. The only difference comes from the weights he attaches to different agents. These weights describe the relative "social" closeness of others to agent 0 , whereas being in subgroup $T$ incorporates the idea of "topological" closeness.

A4 puts restrictions on what can happen inside each subgroup $T$. The rest of the Axioms deal with what happens between different subgroups. Without A5-A6 any two restrictions $\succcurlyeq_{T}$ and $\succcurlyeq_{R}$ are completely unrelated. It is desirable, however, that agent 0 choose somewhat consistently in different subgroups.

For each level of status $x_{0}$ of agent 0 , Axiom A5 asks for the existence of special status level $x^{*}\left(x_{0}\right)$ of any agent $i$, such that agent 0 , when facing the outcome $\left(x_{0}, x^{*}\left(x_{0}\right), h_{-i}\right)$, does not care about $i$ and chooses as if $i$ does not exist. For example, agent 0 might not care about others as long as they have no status or possessions at all $\left(x^{*}\left(x_{0}\right)=0\right)$, but he starts taking them into account once they have more than that.

Axiom A6 requires that there exist two subgroups $T, R \in \mathcal{C}$ to which agent 0 attaches different total social weight. In particular, if for some $\left(x_{0}, x\right)$ it so happens that $\left(x_{0}, x\right)_{T} \dot{\sim}\left(x_{0}, x\right)_{R}$, then if agent 0 prefers having $\left(x_{0}, x^{\prime}\right)_{T}$ to $\left(x_{0}, x\right)_{T}$ then he prefers it also over $\left(x_{0}, x^{\prime}\right)_{R}$. This means that subgroup $T$ is preferable to subgroup 
$R$ only because agent 0 likes having agents $T$ around more than agents $R$. A counterexample might be the situation when all subgroups in $\mathcal{C}$ have the same number of others and the same social weights are attached to all of them. In this case, we will have $h_{T} \dot{\sim} h_{R}$ for all $h \in X_{T}$ and any $T, R \in \mathcal{C}$, which leads to the indeterminacy of status component of the preferences. Axiom A6 is necessary when there are no two subgroups in $\mathcal{C}$ such that one is the strict subset of the other. If such subgroups exist, then A6 can be dropped without consequences.

Definition 6 Let $f: X \rightarrow \mathbb{R}$ be any function, $u: X^{2} \rightarrow \mathbb{R}$ be status function, and let $\left(\pi_{i}\right)_{i \in S}$ be positive numbers. For $T \in \mathcal{C}$, let $U_{T}: X_{T} \rightarrow \mathbb{R}$ be defined as

$$
U_{T}[h]=E_{h}\left[f\left(x_{0}\right)+\sum_{i \in T} \pi_{i} u\left(x_{0}, x_{i}\right)\right]
$$

Finally, let $U: \mathcal{A} \rightarrow \mathbb{R}$ be equal to $U_{T}[h]$ for all $T \in \mathcal{C}$ and $h \in X_{T}$.

Theorem 4 The following two statements are equivalent:

1. $\succcurlyeq$ satisfies $A 0-A 6$

2. $\succcurlyeq$ has a utility representation $U: \mathcal{A} \rightarrow \mathbb{R}$ as described in Definition 6, unique up to a positive affine transformation.

Proof See Sect. 7.

\section{Discussion}

\subsection{Group size matters}

In this subsection, I discuss why it is intuitive to assume that people have preferences over the group they belong to. In many theoretical and empirical papers which include social preferences (Bolton and Ockenfels 2000; Luttmer 2005), it is assumed that agents care about their own possessions and the mean of the distribution of the possessions of others. This assumption implies that the size or the identity of the group to which the agent compares herself become irrelevant. There are at least two good reasons why the agent might care about the size or the identity of the reference group.

The first reason is that larger groups of people will most likely have more diverse distribution of skills. Thus, being average among some big group of others should not be indifferent to being average among the group's subset. Suppose that the game theorist from the previous example has average number of publications among people on her department (which is, say, ranked 100). It seems very plausible that when she is asked a question "Do you prefer to have average number of top publications among people on your department or average number of top publications among all economists?" she would opt for the latter. The same idea can be applied to any environment in which agents are competing with each other. For example, in soccer players care about in which league their team is playing. They would definitely prefer to be in average team of the first league rather than second. In general, the same effect should be observed in environments where people care about their reputation: it would 
be desirable for people with good (bad) reputation to have more (less) others know about it.

The second reason is that if the agent cares only about the mean of others' possessions it is impossible to separate consumption from status for the reasons described in the beginning of this section. Given fixed possession of the agent and the distribution of possessions of others, the agent is indifferent which group to belong to. This removes the leverage that allows to uniquely separate consumption and status.

\section{Proofs}

Proof of Theorem 1 Theorem 2 shows that $\succcurlyeq$ can be represented by expected utility functions $V_{T}\left(x_{0}, x\right)=g\left(x_{0}\right)+v_{T}\left(x_{0}, x\right)$ where $v_{T}\left(x_{0}, x\right)$ are status functions satisfying Definition 3. By E6, for any $x_{0} \in X$ either 1) $v_{T}\left(x_{0}, \cdot\right) \equiv 0$ or 2) there are $x, x^{\prime} \in X$ with $v_{T}\left(x_{0}, x\right)>0>v_{T}\left(x_{0}, x^{\prime}\right)$. Consider any $x_{0}$ for which the latter holds. Let $\beta^{*}$ be such that $\beta^{*} v_{S_{2}}\left(x_{0}, x\right)+\left(1-\beta^{*}\right) v_{S_{2}}\left(x_{0}, x^{\prime}\right)=0$. Then by E5

$$
\begin{aligned}
& \beta \in\left[0, \beta^{*}\right) \Rightarrow \beta v_{S_{1}}\left(x_{0}, x\right)+(1-\beta) v_{S_{1}}\left(x_{0}, x^{\prime}\right)<\beta v_{S_{2}}\left(x_{0}, x\right)+(1-\beta) v_{S_{2}}\left(x_{0}, x^{\prime}\right)<0 \\
& \beta \in\left(\beta^{*}, 1\right] \Rightarrow \beta v_{S_{1}}\left(x_{0}, x\right)+(1-\beta) v_{S_{1}}\left(x_{0}, x^{\prime}\right)>\beta v_{S_{2}}\left(x_{0}, x\right)+(1-\beta) v_{S_{2}}\left(x_{0}, x^{\prime}\right)>0
\end{aligned}
$$

This implies that

$$
\begin{aligned}
& \lim _{\beta \uparrow \beta^{*}} \beta v_{S_{1}}\left(x_{0}, x\right)+(1-\beta) v_{S_{1}}\left(x_{0}, x^{\prime}\right) \leq 0 \\
& \lim _{\beta \downarrow \beta^{*}} \beta v_{S_{1}}\left(x_{0}, x\right)+(1-\beta) v_{S_{1}}\left(x_{0}, x^{\prime}\right) \geq 0 .
\end{aligned}
$$

Since $\beta v_{S_{1}}\left(x_{0}, x\right)+(1-\beta) v_{S_{1}}\left(x_{0}, x^{\prime}\right)$ is a continuous function of $\beta$, the only way both inequalities can hold is if $\beta^{*} v_{S_{1}}\left(x_{0}, x\right)+\left(1-\beta^{*}\right) v_{S_{1}}\left(x_{0}, x^{\prime}\right)=0$. This, together with analogous equality for $v_{S_{2}}$ lets us conclude that

$$
\frac{v_{S_{2}}\left(x_{0}, x^{\prime}\right)}{v_{S_{1}}\left(x_{0}, x^{\prime}\right)}=\frac{v_{S_{2}}\left(x_{0}, x\right)}{v_{S_{1}}\left(x_{0}, x\right)}=: \pi\left(x_{0}\right) \in(0,1)
$$

This definition of $\pi\left(x_{0}\right)$ is unambiguous since the above equality holds for any $x$ and $x^{\prime}$ for which $v_{T}\left(x_{0}, x\right)>0>v_{T}\left(x_{0}, x^{\prime}\right)$. Therefore, $v_{S_{1}}\left(x_{0}, x\right)=\pi\left(x_{0}\right) v_{S_{2}}\left(x_{0}, x\right)$.

In the other case, when $v_{T}\left(x_{0}, \cdot\right) \equiv 0$ set $\pi\left(x_{0}\right)$ to be any number between 0 and 1 .

Proof of Theorem $2[\mathbf{1} \Longrightarrow$ 2] By Theorem 8.4 of Fishburn (1970), Axioms E1-E3 together with mixture set structure on $\Delta\left(X^{2}\right)$ generated by compound lottery rule imply that for all $T \in \mathcal{S}$, preference relation $\succcurlyeq$ restricted to $\mathcal{E}_{T}$ has expected utility representation

$$
h \succcurlyeq z \Longleftrightarrow \bar{V}_{T}[h] \geq \bar{V}_{T}[z]
$$

for some functions $\bar{V}_{T}$, fixed up to a positive affine transformation. 
Let

$$
X^{*}:=\left\{\left(x_{0}, \eta\right) \in \Delta\left(X^{2}\right):\left(x_{0}, \eta\right)_{S_{1}} \sim\left(x_{0}, \eta\right)_{S_{2}}\right\}
$$

and consider the set $\Delta\left(X^{*}\right)$. By E4.1, $\Delta\left(X^{*}\right)$ contains elements of the form $\left(x_{0}, x^{*}\right)$ for any $x_{0} \in X$ and by E5, for all $x_{0} \in X, T \in \mathcal{S}$ and all $\left(x_{0}, \eta\right),\left(x_{0}, \nu\right) \in \Delta\left(X^{*}\right)$, $\left(x_{0}, \eta\right)_{T} \sim\left(x_{0}, v\right)_{T}$. To see that the latter statement holds, suppose, by contradiction, that there are $\left(x_{0}, \eta\right),\left(x_{0}, v\right) \in \Delta\left(X^{*}\right)$ such that $\left(x_{0}, v\right)_{S_{2}} \succ\left(x_{0}, \eta\right)_{S_{2}}$. Then, E5 implies that $\left(x_{0}, v\right)_{S_{1}} \succ\left(x_{0}, v\right)_{S_{2}}$, which contradicts the assumption that $\left(x_{0}, v\right) \in$ $\Delta\left(X^{*}\right)$.

The consequences of E4.1 and E5 imply that for any $T \in \mathcal{S}$ the restriction of $\bar{V}_{T}$ to $\Delta\left(X^{*}\right)$ can be written as

$$
\bar{V}_{T}[h]=E_{h}\left[g_{T}\left(x_{0}\right)\right]
$$

for some function $g_{T}: X \rightarrow \mathbb{R}$. E4.2 implies that for any $\ell \in \Delta\left(X^{*}\right)$ it is true that $\ell_{S_{1}} \sim \ell_{S_{2}}$. Therefore, for any $\ell, m \in \Delta\left(X^{*}\right)$

$$
\ell_{S_{1}} \succcurlyeq m_{S_{1}} \Longleftrightarrow \ell_{S_{2}} \succcurlyeq m_{S_{2}}
$$

since all similar elements in the two copies of $\Delta\left(X^{*}\right)$ are connected by the indifference relation.

This means that the restriction of $\succcurlyeq$ to $\Delta\left(X^{*}\right)$, viewed as a subset of either $\mathcal{E}_{S_{1}}$ or $\mathcal{E}_{S_{2}}$, induces the order, independent of the group. $\Delta\left(X^{*}\right)$ is a mixture set with mixture structure inherited from $\Delta\left(X^{2}\right)$. In addition, properties E1-E3 transfer to $\Delta\left(X^{*}\right)$. Therefore, by Theorem 8.4 of Fishburn (1970), $g_{S_{1}}$ and $g_{S_{2}}$ are positive affine transformations of one another. This makes it possible to apply positive affine transformation to $\bar{V}_{S_{2}}$ obtaining $V_{S_{2}}$ (which still represents $\succcurlyeq$ on $\mathcal{E}_{S_{2}}$ ) so that

$$
\bar{V}_{S_{1}}\left[\left(x_{0}, \eta\right)_{S_{1}}\right]=: V_{S_{1}}\left[\left(x_{0}, \eta\right)_{S_{1}}\right]=V_{S_{2}}\left[\left(x_{0}, \eta\right)_{S_{2}}\right]=: g\left(x_{0}\right)
$$

whenever $\left(x_{0}, \eta\right)_{S_{1}} \sim\left(x_{0}, \eta\right)_{S_{2}}$.

Let $v_{T}\left(x_{0}, x\right):=V_{T}\left(x_{0}, x\right)-g\left(x_{0}\right)$. We can rewrite $V_{T}\left(x_{0}, x\right)=g\left(x_{0}\right)+v_{T}\left(x_{0}, x\right)$. Condition (4.2) for functions $v_{T}$ is satisfied since the set $X^{*}$ contains all elements $\left(x_{0}, x^{*}\right)$ for which agent 0 is indifferent between the two groups. Conditions (4.3) and (4.4) follow from E5.

[2 $\Longrightarrow 1$ 1] E1-E3 follow from the only if part of Theorem 8.4 of Fishburn (1970). E4.1 is a consequence of $v_{S_{1}}$ and $v_{S_{2}}$ being status functions. E4.2 is obvious. E5 directly follows from the properties (4.2-4.4).

Proof of Theorem $3[\mathbf{1} \Longrightarrow 2$ ] By the same argument as in the proof of Theorem 2, we can construct the utility representation $h \succcurlyeq z \Longleftrightarrow \bar{V}_{T}[h] \geq \bar{V}_{T}[z]$ for some functions $\bar{V}_{T}$, fixed up to a positive affine transformation. Let $\Delta\left(X^{*}\right)$ be as in the proof of Theorem 2, then by E4.1, $\Delta\left(X^{*}\right)$ contains elements of the form $\left(x_{0}, x\right)$ for any $x_{0} \in X$ and by $\mathrm{E}^{\star}$, for all $x_{0} \in X, T \in \mathcal{S}$ and all 
$\left(x_{0}, \eta\right),\left(x_{0}, v\right) \in \Delta\left(X^{*}\right),\left(x_{0}, \eta\right)_{T} \sim\left(x_{0}, v\right)_{T}$. To see that the latter statement holds, consider any $\left(x_{0}, \eta\right),\left(x_{0}, v\right) \in \Delta\left(X^{*}\right)$. Then by E5 $5^{\star}$ and the construction of $\Delta\left(X^{*}\right)$

$$
\left(\pi\left(x_{0}, \eta\right)+(1-\pi)\left(x_{0}, v\right)\right)_{S_{1}} \sim\left(x_{0}, \eta\right)_{S_{2}} \sim\left(x_{0}, \eta\right)_{S_{1}} .
$$

This implies that $\left(x_{0}, v\right)_{S_{1}} \sim\left(x_{0}, \eta\right)_{S_{1}}$ since otherwise Independence Axiom (E2) would be violated. $\left(x_{0}, v\right)_{S_{2}} \sim\left(x_{0}, \eta\right)_{S_{2}}$ now follows from the transitivity of $\sim$.

Now, by the same argument, definitions and notation as in the proof of Theorem 2 we can rewrite $V_{T}\left(x_{0}, x\right)=g\left(x_{0}\right)+v_{T}\left(x_{0}, x\right)$. By E5 ${ }^{\star}$, there exists $\pi \in(0,1)$ such that for all $x_{0}, x \in X$

$$
\pi\left(g\left(x_{0}\right)+v_{S_{1}}\left(x_{0}, x\right)\right)+(1-\pi) g\left(x_{0}\right)=g\left(x_{0}\right)+v_{S_{2}}\left(x_{0}, x\right) .
$$

This implies $\pi v_{S_{1}}\left(x_{0}, x\right)=v_{S_{2}}\left(x_{0}, x\right)=: \pi v\left(x_{0}, x\right)$, which is exactly the requirement of Definition 4. The functions $v$ and $\pi v$ are status functions by E4.1.

[2 $\Longrightarrow 1$ 1] E1-E3 follow from the only if part of Theorem 8.4 of Fishburn (1970). E4. 1 is a consequence of $v$ being status functions. E4.2 is obvious. E5 ${ }^{\star}$ directly follows from the property (4.5).

Proof of Theorem $4[\mathbf{1} \Longrightarrow$ 2] The idea of the proof is to establish the existence of the weighted-additive utilities $U_{T}$ for all $T \in \mathcal{C}$, then show that a unique function $f$ can be constructed in a way that is consistent with each of the utility functions, and, finally, rescale the now redefined utility functions to show that all the utilities can have the specific form described in the Theorem.

1. Fix any $T \in \mathcal{C}$. Then $\mathrm{A} 1-\mathrm{A} 3$ and the fact that $\mathcal{X}_{T}$ is a mixture set imply the existence of the expected utility $U_{T}: X_{T} \rightarrow \mathbb{R}$, unique up to a positive affine transformation (Theorem 8.4 of Fishburn (1970)). Lemma 1 shows that $U_{T}$ is the weighted-additive expected utility:

$$
U_{T}[h]=\sum_{i \in T} \pi_{T}^{i} E_{h}\left[\bar{u}_{T}\left(x_{0}, x\right)\right]
$$

where $\pi_{T}^{i}>0$.

2. Lemma 3 says that for any $x_{0}$ there exists a non-empty set $X_{x_{0}}^{*}$ which consists of all the points $x \in X_{x_{0}}^{*}$ such that for all $T, R \in \mathcal{C}\left(x_{0}, x\right)_{T} \dot{\sim}\left(x_{0}, x\right)_{R}$. Moreover, for all $x, y \in X_{x_{0}}^{*}$ and all $T \in \mathcal{C}$ we have $\left(x_{0}, x\right)_{T} \dot{\sim}\left(x_{0}, y\right)_{T} .{ }^{19}$ The pairs in $X_{x_{0}}^{*}$ are perfect candidates for the representation of the pure consumption value of $x_{0}$ : agent 0 does not care to which subgroup he belongs when choosing among pairs from sets $X_{x_{0}}^{*}$. Let

$$
X^{*}:=\bigcup_{x_{0} \in X}\left\{\left(x_{0}, x\right) \in X^{2}: x \in X_{x_{0}}^{*}\right\} .
$$

\footnotetext{
19 Lemma 3 makes sure that there are no other points outside $X_{x_{0}}^{*}$ that satisfy these conditions. Thus, $X_{x_{0}}^{*}$ is the biggest "unique" set with these properties.
} 
Notice that the choice between any two pairs $\left(x_{0}, x\right),\left(y_{0}, y\right) \in X^{*}$ depends only on $x_{0}$ and $y_{0}$ and nothing else. In terms of the utilities defined on the previous step, we have

$$
U_{T}\left[\left(x_{0}, x\right)_{T}\right]=U_{T}\left[\left(x_{0}, y\right)_{T}\right]=U_{R}\left[\left(x_{0}, x\right)_{R}\right]
$$

for all $\left(x_{0}, x\right),\left(x_{0}, y\right) \in X^{*}$, all $T, R \in \mathcal{C}$.

Define $f: X \rightarrow \mathbb{R}$ to be

$$
f\left(x_{0}\right):=U_{T}\left[\left(x_{0}, x\right)_{T}\right]
$$

for any $x \in X_{x_{0}}^{*}$ and any $T \in \mathcal{C}$ and rewrite $U_{T}$ as

$$
U_{T}\left[\left(x_{0},\left(x_{i}\right)_{i \in T}\right)\right]=f\left(x_{0}\right)+\sum_{i \in T} \pi_{T}^{i} u_{T}\left(x_{0}, x_{i}\right)
$$

where $u_{T}\left(x_{0}, x\right)=\bar{u}_{T}\left(x_{0}, x\right)-f\left(x_{0}\right) / \sum_{i} \pi_{T}^{i}$ and $u_{T}\left(x_{0}, x\right)=0$ for all $\left(x_{0}, x\right) \in$ $X^{*}$.

3. Fix $i \in S$ and consider all subgroups $C_{1}, \ldots, C_{k} \in \mathcal{C}$ to which $i$ belongs. Then A5 with $Q=\{i\}$ implies that for all $\left(x_{0}, x\right) \in X^{2}$

$$
\left(x_{0}, x_{i},\left(x^{*}\left(x_{0}\right)\right)_{-i}\right)_{C_{1}} \dot{\sim} \ldots \dot{\sim}\left(x_{0}, x_{i},\left(x^{*}\left(x_{0}\right)\right)_{-i}\right)_{C_{k}}
$$

Therefore,

$$
f\left(x_{0}\right)+\pi_{C_{1}}^{i} u_{C_{1}}\left(x_{0}, x\right)=\cdots=f\left(x_{0}\right)+\pi_{C_{k}}^{i} u_{C_{k}}\left(x_{0}, x\right)
$$

implying

$$
\pi_{C_{1}}^{i} u_{C_{1}}\left(x_{0}, x\right)=\pi_{C_{2}}^{i} u_{C_{2}}\left(x_{0}, x\right)=\cdots=\pi_{C_{k}}^{i} u_{C_{k}}\left(x_{0}, x\right)
$$

for all $\left(x_{0}, x\right) \in X^{2}$.

Now fix some $T, R \in \mathcal{C}$ such that there are $i, j \in T \cap R$. Then by the above

$$
\begin{aligned}
& \pi_{T}^{i} u_{T}\left(x_{0}, x\right)=\pi_{R}^{i} u_{R}\left(x_{0}, x\right) \\
& \pi_{T}^{j} u_{T}\left(x_{0}, x\right)=\pi_{R}^{j} u_{R}\left(x_{0}, x\right)
\end{aligned}
$$

By A1, the preferences $\succcurlyeq$ are non-trivial on all subgroups. So, there is $\left(y_{0}, y\right) \in X^{2}$ such that $u_{T}\left(y_{0}, y\right) \neq 0$. The connectedness of $\mathcal{C}$, positiveness of $\left(\pi_{T}^{i}\right)$ and (7.2) implies then that $u_{C}\left(y_{0}, y\right) \neq 0$ for all $C \in \mathcal{C}$.

The Eqs. 7.3-7.4 hold for $\left(y_{0}, y\right)$. So, by dividing them we obtain

$$
\frac{\pi_{T}^{i}}{\pi_{R}^{i}}=\frac{\pi_{T}^{j}}{\pi_{R}^{j}}=: L_{T, R}
$$

for all intersecting $T, R$ and all $i, j \in T \cap R$. If $T \cap R$ has only one element $i$, then set 


$$
\frac{\pi_{T}^{i}}{\pi_{R}^{i}}=: L_{T, R}
$$

Notice as well that for $L>0$

$$
f\left(x_{0}\right)+\sum_{i \in T} \pi_{T}^{i} u_{T}\left(x_{0}, x_{i}\right)=f\left(x_{0}\right)+\sum_{i \in T} \frac{\pi_{T}^{i}}{L}\left(L u_{T}\left(x_{0}, x_{i}\right)\right)
$$

For intersecting $T, R$, we can rescale all the weights $\left(\pi_{T}^{i}\right)$ and $u_{T}$ using $L_{T, R}$ in place of $L$ in (7.6). This makes the weights for all $i \in T \cap R$ equal in both subgroups. Also, rescaled $u_{T}$ becomes equal to $u_{R}$. Denote this rescaled $U_{T}$ by $L_{T, R}\left(U_{T}\right)$.

4. $\mathcal{C}$ can be represented as a graph. Let all elements of $\mathcal{C}$ be nodes. Two nodes $C_{1}, C_{2}$ are connected by an edge if $C_{1} \cap C_{2} \neq \emptyset$. By definition of $\mathcal{C}$, the resulting finite graph $G$ is connected. ${ }^{20}$

For each node $C \in G$, there corresponds a collection of weights $\left(\pi_{C}^{i}\right)$ and a status function $u_{C}$. Call $\left\langle G,\left\{\left(\pi_{C}^{i}\right), u_{C}\right\}_{C \in G}\right\rangle$ a graph structure.

Choose any nodes $\left(T,\left(\pi_{T}^{i}\right), u_{T}\right)$ and $\left(R,\left(\pi_{R}^{i}\right), u_{R}\right)$ connected by an edge. Rescale $U_{T}$ to $L_{T, R}\left(U_{T}\right)$ and contract the two nodes into one node $\left(T \cup R,\left(\pi_{T \cup R}^{i}\right), u_{T \cup R}\right)$, where $u_{T \cup R}=u_{R}$.

This turns the structure $\left\langle G,\left\{\left(\pi_{C}^{i}\right), u_{C}\right\}_{C \in G}\right\rangle$ into the structure

$$
\left\langle G_{1},\left\{\left(\left(\pi_{T \cup R}^{i}\right), u_{T \cup R}\right),\left(\left(\pi_{C}^{i}\right), u_{C}\right)\right\}_{C \in G \backslash\{T, R\}}\right\rangle
$$

where $G_{1}$ is a minor of $G$ obtained by the contraction of an edge between $T$ and $R$.

Continue contracting edges until there are none left. The sequence of graph structures thus obtained is finite and its last element $\left\langle G_{N},\left(\pi_{S}^{i}\right), u_{S}\right\rangle$ has one node and no edges. By construction, for any agent $i \in S$ the weight $\pi_{S}^{i}$ is the same in all subgroups $i$ belongs to. The status function $u_{S}$ is also same in all subgroups. Let $\pi_{i}=\pi_{S}^{i}$ and $u=u_{S}$, then we obtain desired utility $U: \mathcal{A} \rightarrow \mathbb{R}$ defined on $x_{T}$ as

$$
U_{T}[h]=E_{h}\left[f\left(x_{0}\right)+\sum_{i \in T} \pi_{i} u\left(x_{0}, x_{i}\right)\right]
$$

Each $U_{T}$ is unique up to a positive affine transformation. In addition, all functions $U_{T}$ are restricted by A5 (AF5) to have the same weights and status functions. Thus, the whole $U$ is unique up to a positive affine transformation.

[2 $\Longrightarrow 1$ ] A1 holds since $u$ is a status function, which is assumed to be not constant. For any $T \in \mathcal{C}, \mathrm{A} 2-\mathrm{A} 3$ hold by the "only if" part of the Theorem 8.4 of Fishburn (1970). Additivity of $U_{T}$ immediately implies A0 and A4. The assumption that $u$ is a

20 See the definitions of all graph theoretic terms in Diestel (2000). 
status function implies that for each $x_{0}$ there is $x^{*}\left(x_{0}\right)$ such that $u\left(x_{0}, x^{*}\left(x_{0}\right)\right)=0$, so A5 follows. It is left to show that A6 holds. Without loss of generality assume that

$$
\sum_{i \in S_{1}} \pi_{i}>\sum_{i \in S_{2}} \pi_{i}
$$

where $S_{1}$ and $S_{2}$ are as in the description of this Theorem. Suppose for some $\left(x_{0}, x\right) \in$ $X^{2}$ we have $U\left[\left(x_{0}, x\right)_{S_{1}}\right]=U\left[\left(x_{0}, x\right)_{S_{2}}\right]$. Then

$$
\sum_{i \in S_{1}} \pi_{i} u\left(x_{0}, x\right)=\sum_{i \in S_{2}} \pi_{i} u\left(x_{0}, x\right)
$$

can happen only when $u\left(x_{0}, x\right)=0$. Now, take any $x^{\prime}$ such that $U\left[\left(x_{0}, x^{\prime}\right)_{S_{1}}\right]>$ $U\left[\left(x_{0}, x\right)_{S_{1}}\right]$. This implies that $u\left(x_{0}, x^{\prime}\right)>u\left(x_{0}, x\right)=0$. But then

$$
\sum_{i \in S_{1}} \pi_{i} u\left(x_{0}, x^{\prime}\right)>\sum_{i \in S_{2}} \pi_{i} u\left(x_{0}, x^{\prime}\right)
$$

and therefore $U\left[\left(x_{0}, x^{\prime}\right)_{S_{1}}\right]>U\left[\left(x_{0}, x^{\prime}\right)_{S_{2}}\right]$. This is the first part of A6. Second part is proved by the exactly same argument.

\section{Lemmata}

Lemma 1 Suppose that $\succcurlyeq$ satisfies $A 0-A 4$. Then for any $T \in \mathcal{C}$, preference relation $\succcurlyeq$ restricted to $X_{T}$ has expected utility representation of the form

$$
U_{T}[h]=\sum_{i \in T} \pi_{T}^{i} E_{h}\left[\bar{u}_{T}\left(x_{0}, x_{i}\right)\right]
$$

where $\pi_{T}^{i}>0$ for all $i \in T$. Moreover, $U_{T}$ is unique up to a positive affine transformation.

Proof Fix any $T \subseteq \mathcal{C}$ and consider the restriction of $\succcurlyeq$ to $\mathcal{X}_{T}$. A1-A3 hold for $\succcurlyeq$ on $X_{T}$. Standard compound lotteries rule turns $X_{T}$ into a mixture set. Thus, by Theorem 8.4 of Fishburn (1970), $\succcurlyeq$ on $X_{T}$ has expected utility representation

$$
U_{T}[h]=E_{h}\left[\bar{u}_{T}\left(x_{0},\left(x_{i}\right)_{i \in T}\right)\right] .
$$

unique up to a positive affine transformation. ${ }^{21}$

Now fix any $x_{0} \in X$ and consider the set

$$
X_{x_{0}}:=\left\{\left(x_{0}, p\right): p \in \Delta\left(X^{T}\right)\right\} .
$$

\footnotetext{
21 I abuse notation by having two functions $\bar{u}_{T}\left(x_{0}, x_{i}\right)$ and $\bar{u}_{T}\left(x_{0},\left(x_{i}\right)_{i \in T}\right)$ with the same name but different arguments. It is always clear from the exposition which function is considered.
} 
Let $\sim_{*}$ be the equivalence relation on $X_{x_{0}}$ defined by

$$
\left(x_{0}, p\right) \sim_{*}\left(x_{0}, q\right) \Leftrightarrow \Pi \mu_{i}(p)=\Pi \mu_{i}(q) .
$$

Let

$$
M_{x_{0}}:=X_{x_{0}} / \sim_{*}
$$

be the set of equivalence classes of $\sim_{*} . M_{x_{0}}$ can be described as

$$
M_{x_{0}}=\left\{\left(\eta_{i}\right)_{i \in T}: \eta_{i} \in \Delta(X)\right\}=\Delta^{T}(X)
$$

where element $\left(\eta_{i}\right)_{i \in T}$ corresponds to $\left(x_{0}, \Pi \eta_{i}\right) \in X_{x_{0}}$. Let us also use notation $\Pi \mu_{i}(p) \in M_{x_{0}}$ to emphasize that $\Pi \mu_{i}(p)=\left(\mu_{i}(p)\right)_{i \in T}$ is the equivalence class of $\left(x_{0}, p\right) \in X_{x_{0}}$.

Notice that by A0 $h \sim_{*} z \Rightarrow h \dot{\sim} z$. Thus, we can view $M_{x_{0}}$ as (almost) a set of equivalence classes of $\dot{\sim}$ with some elements still being indifferent under $\dot{\sim}{ }^{22}$ It is therefore natural to extend $\succcurlyeq$ to $M_{x_{0}}$ by setting

$$
\left(\eta_{i}\right)_{i \in T} \succcurlyeq\left(v_{i}\right)_{i \in T} \Leftrightarrow\left(x_{0}, \Pi \eta_{i}\right) \succcurlyeq\left(x_{0}, \Pi v_{i}\right)
$$

The set $M_{x_{0}}$ also naturally inherits the mixture set structure from $X_{x_{0}}$ (which inherits it from $X_{T}$ ) by associating the mixture of two indifference classes with the indifference class of the mixture of any two elements inside those indifference classes. $^{23}$ This mixture set structure will play the pivotal role in the proof, thus it is necessary to show first that this procedure indeed generates mixture set structure on $M_{x_{0}}$.

Define a mixture on $M_{x_{0}}$ by

$$
\alpha \Pi \mu_{i}(p)+(1-\alpha) \Pi \mu_{i}(q)=\Pi \mu_{i}(\alpha p+(1-\alpha) q)
$$

The first task is to show that this definition is independent of the choice of $p$ and $q$ : any $p^{\prime}$ with $\Pi \mu_{i}\left(p^{\prime}\right)=\Pi \mu_{i}(p)$ and $q^{\prime}$ with $\Pi \mu_{i}\left(q^{\prime}\right)=\Pi \mu_{i}(q)$, used in the above definition, should generate the same mixture. Indeed, it is a straightforward property of marginal distributions that

$$
\mu_{i}(\alpha p+(1-\alpha) q)=\alpha \mu_{i}(p)+(1-\alpha) \mu_{i}(q)
$$

Therefore,

$$
\alpha \Pi \mu_{i}(p)+(1-\alpha) \Pi \mu_{i}(q)=\Pi\left(\alpha \mu_{i}(p)+(1-\alpha) \mu_{i}(q)\right) .
$$

\footnotetext{
${ }^{22} X_{x_{0}} / \dot{\sim}$ is a partition of $X_{x_{0}} . M_{x_{0}}$ is weakly finer partition.

${ }^{23}$ Mixture set structure on some set $Z$ is a mapping $Z^{2} \times[0,1] \mapsto Z$ which defines a "mixture" of two elements with coefficient $\alpha \in[0,1]$ in $Z$. The mapping satisfies simple Axioms like associativity and compound lottery rule (Herstein and Milnor 1953).
} 
This formula depends only on individual marginals $\mu_{i}(p)$ and $\mu_{i}(q)$ and thus is the same for any choice of $p^{\prime}$ and $q^{\prime}$.

The second task is to verify that the mixtures thus defined satisfy the definition of mixture set structure. It is trivial to see that all the assumptions are satisfied (see Definition 8.3 of Fishburn (1970)) since mixture is defined as a function of a mixture of simple lotteries $\left(p, q \in \Delta\left(X^{T}\right)\right)$ which does possess the mixture set structure.

The resulting formula for the mixture has one important property. When $M_{x_{0}}$ is viewed as a product space $\Delta^{T}(X)$ the definition is essentially a component-wise mixture:

$$
\alpha\left(\eta_{i}\right)_{i \in T}+(1-\alpha)\left(v_{i}\right)_{i \in T}=\left(\alpha \eta_{i}+(1-\alpha) v_{i}\right)_{i \in T},
$$

which gives us all the ingredients to use additive utility representation result like Theorem 13.1 of Fishburn (1970). At first, $M_{x_{0}}$ is a component-wise mixture set. At second, $\succcurlyeq$ on $M_{x_{0}}$ inherits the properties A1-A3 of $\succcurlyeq$ on $X_{x_{0}}$, thus satisfying all the requirements of Theorem 13.1 of Fishburn (1970). ${ }^{24}$ This is easy to see since preferences and mixing on $M_{x_{0}}$ were defined through their counterparts on $X_{x_{0}}$. Therefore, any properties of preferences are transferred. All this implies that $\succcurlyeq$ on $M_{x_{0}}$ can be represented by an additive expected utility $\sum_{i \in T} u_{T}^{i}\left(x_{0}, x_{i}\right)$ :

$$
\left(\eta_{i}\right)_{i \in T} \succcurlyeq\left(v_{i}\right)_{i \in T} \Leftrightarrow \sum_{i \in T} E_{\eta_{i}}\left[u_{T}^{i}\left(x_{0}, x\right)\right] \geq \sum_{i \in T} E_{v_{i}}\left[u_{T}^{i}\left(x_{0}, x\right)\right],
$$

unique up to a positive affine transformation. ${ }^{25}$

Since $\succcurlyeq$ on $M_{x_{0}}$ and $\succcurlyeq$ on $X_{x_{0}}$ are essentially the same orders, we can define the utility of $\succcurlyeq$ on $X_{x_{0}}$ by

$$
\left(x_{0}, p\right) \succcurlyeq\left(x_{0}, q\right) \Leftrightarrow \sum_{i \in T} E_{\mu_{i}(p)}\left[u_{T}^{i}\left(x_{0}, x\right)\right] \geq \sum_{i \in T} E_{\mu_{i}(q)}\left[u_{T}^{i}\left(x_{0}, x\right)\right] .
$$

This means that if A0-A3 hold, then it is possible to represent preferences restricted to $X_{x_{0}}$ for any $x_{0}$ by an additive expected utility function. $X_{x_{0}}$ is a mixture set and thus by the only if part of Theorem 8.4 of Fishburn (1970) any utility that represents $\succcurlyeq$ on $X_{x_{0}}$ is a positive affine transformation of any other utility. This implies, in particular, that representation $\bar{u}_{T}\left(x_{0},\left(x_{i}\right)_{i \in T}\right)$ for $\succcurlyeq$ on $x_{T}$ obtained in the beginning of this proof should be positive affine transformation of some additive utility function for any $x_{0}$, since $\bar{u}_{T}\left(x_{0},\left(x_{i}\right)_{i \in T}\right)$ restricted to any $X_{x_{0}}$ still represent $\succcurlyeq$ on it. Therefore, for all $x_{0}$, it is possible to write

\footnotetext{
24 To verify that $\succ$ is a weak order see Proposition 2.4 of Kreps (1988).

25 The expectation in this expression is taken with respect to the second argument of the functions $u_{T}^{i}\left(x_{0}, x\right)$. The first argument is fixed.
} 


$$
\begin{aligned}
\bar{u}_{T}\left(x_{0},\left(x_{i}\right)_{i \in T}\right) & =a_{x_{0}} \sum_{i \in T} u_{T}^{i}\left(x_{0}, x_{i}\right)+b_{x_{0}}=\sum_{i \in T}\left(a_{x_{0}} u_{T}^{i}\left(x_{0}, x_{i}\right)+\frac{b_{x_{0}}}{|T|}\right) \\
& =\sum_{i \in T} \bar{u}_{T}^{i}\left(x_{0}, x_{i}\right)
\end{aligned}
$$

where $a_{x_{0}}>0$ and $b_{x_{0}}$ is some number.

To finish the proof of this Lemma it is left to show that all functions $\bar{u}_{T}^{i}$ are multiples of one another. We use A4 to achieve that. Indeed, by A4, for all $i, j \in T$ and any $\ell, m \in \Delta\left(X^{2}\right)$ it is true that

$$
E_{\ell}\left[\bar{u}_{T}^{i}\left(x_{0}, x\right)\right] \geq E_{m}\left[\bar{u}_{T}^{i}\left(x_{0}, x\right)\right] \Leftrightarrow E_{\ell}\left[\bar{u}_{T}^{j}\left(x_{0}, x\right)\right] \geq E_{m}\left[\bar{u}_{T}^{j}\left(x_{0}, x\right)\right],
$$

which can happen only if $\bar{u}_{T}^{i}$ and $\bar{u}_{T}^{j}$ are positive affine transformations of one another. This implies that for all $i \in T$ we can write

$$
\bar{u}_{T}^{i}\left(x_{0}, x\right)=a_{i} \bar{u}_{T}^{1}\left(x_{0}, x\right)+b_{i}
$$

where $\{1\} \in T, a_{i}>0$ and $b_{i}$ is some number. Let $\bar{u}_{T}\left(x_{0}, x\right)=\bar{u}_{T}^{1}\left(x_{0}, x\right)+$ $\sum_{i} b_{i} / \sum_{i} a_{i}$ and denote $\pi_{T}^{i}=a_{i}$. Then

$$
\bar{u}_{T}^{i}\left(x_{0}, x\right)=\sum_{i \in T} \pi_{T}^{i} \bar{u}_{T}\left(x_{0}, x_{i}\right)
$$

and $U_{T}[h]=\sum_{i \in T} \pi_{T}^{i} E_{h}\left[\bar{u}_{T}\left(x_{0}, x_{i}\right)\right]$ represents $\succcurlyeq$ as desired.

Lemma 2 Suppose A5-A6 hold. Then for all $C_{1}, C_{2} \in \mathcal{C}$, all $x_{0} \in X$ and all $x^{*}\left(x_{0}\right) \in X$ satisfying A5

$$
\left(x_{0}, x^{*}\left(x_{0}\right)\right)_{C_{1}} \dot{\sim}\left(x_{0}, x^{*}\left(x_{0}\right)\right)_{C_{2}}
$$

Proof Let us first assume that $C_{1} \cap C_{2} \neq \emptyset$. Then, by putting $x=x^{*}\left(x_{0}\right)$ in the definition of A5, we get the desired

$$
\left(x_{0}, x^{*}\left(x_{0}\right)\right)_{C_{1}} \dot{\sim}\left(x_{0}, x^{*}\left(x_{0}\right)\right)_{C_{2}}
$$

Now, $\mathcal{C}$ is the connected collection of subsets (see Definition 5). Therefore, any two disjoint subgroups can be connected by the sequence of intersecting ones. Therefore, by transitivity of $\dot{\sim}$ the result above holds for all $C_{1}, C_{2} \in \mathcal{C}$.

Lemma 3 Suppose A5-A6 hold. Then for all $x_{0} \in X$, there exists a non-empty set

$$
X_{x_{0}}^{*}=\left\{x \in X: \forall T, R \in \mathcal{C}\left(x_{0}, x\right)_{T} \dot{\sim}\left(x_{0}, x\right)_{R}\right\} .
$$

Moreover, for all $x_{0} \in X, x, y \in X_{x_{0}}^{*}$ and all $T \in \mathcal{C}$

$$
\left(x_{0}, x\right)_{T} \dot{\sim}\left(x_{0}, y\right)_{T}
$$


Proof A5 says that for all $x_{0} \in X$ there is $x^{*}\left(x_{0}\right)$, which by Lemma 2 satisfies the condition for being a member of $X_{x_{0}}^{*}$. Therefore, we have shown that non-empty $X_{x_{0}}^{*}$ exists for all $x_{0}$.

Now suppose that the second condition of the Lemma does not hold. In other words, there is $x_{0}$ and $x, y \in X_{x_{0}}^{*}$ such that for some $T \in \mathcal{C}$

$$
\left(x_{0}, x\right)_{T} \succ\left(x_{0}, y\right)_{T}
$$

Let $S_{1}, S_{2} \in \mathcal{C}$ be the two subgroups satisfying A6. Then, by definition of $y$

$$
\left(x_{0}, y\right)_{S_{1}} \dot{\sim}\left(x_{0}, y\right)_{S_{2}}
$$

Moreover, the definitions of $x$ and $y$ and the assumption give

$$
\left(x_{0}, x\right)_{S_{1}} \dot{\sim}\left(x_{0}, x\right)_{T} \dot{\succ}\left(x_{0}, y\right)_{T} \dot{\sim}\left(x_{0}, y\right)_{S_{1}}
$$

The two conditions above and A6 imply that

$$
\left(x_{0}, x\right)_{S_{1}} \dot{\succ}\left(x_{0}, x\right)_{S_{2}}
$$

which contradicts the fact that $x$ is an element of $X_{x_{0}}^{*}$.

Acknowledgments The author would like to thank Aldo Rustichini, Marcel K. Richter, and Michele Boldrin for innumerable conversations and guidance that shaped his comprehension of the subject and resulted in this article. The author is grateful to Andrew Cassey, Joshua Miller, Philipp Reiss, Arno Riedl, and the participants of the Neuroeconomics workshop at the University of Minnesota for insightful comments. All mistakes are his.

Open Access This article is distributed under the terms of the Creative Commons Attribution Noncommercial License which permits any noncommercial use, distribution, and reproduction in any medium, provided the original author(s) and source are credited.

\section{Appendix}

Theorem 2 with modified E5

$\mathbf{E 5}^{\bullet}$ For all $x_{0} \in X$ and $\eta, v \in \Delta(X)$

$$
\left[\left(x_{0}, \eta\right)_{S_{1}} \sim\left(x_{0}, \eta\right)_{S_{2}} \wedge\left(x_{0}, v\right)_{S_{1}} \sim\left(x_{0}, v\right)_{S_{2}}\right] \Longrightarrow\left(x_{0}, \eta\right)_{S_{1}} \sim\left(x_{0}, v\right)_{S_{1}}
$$

Definition 7 Let $g: X \rightarrow \mathbb{R}$ be any function, and $v_{S_{1}}, v_{S_{2}}: X^{2} \rightarrow \mathbb{R}$ be status functions such that for any $x_{0}, x \in X$

$$
v_{S_{1}}\left(x_{0}, x\right)=v_{S_{2}}\left(x_{0}, x\right) \Longleftrightarrow v_{S_{1}}\left(x_{0}, x\right)=0
$$

For $T \in \mathcal{S}$, let $V_{T}: \mathcal{E}_{T} \rightarrow \mathbb{R}$ be defined as

$$
V_{T}[h]=E_{h}\left[g\left(x_{0}\right)+v_{T}\left(x_{0}, x\right)\right] .
$$


Finally, let $V: \mathcal{E} \rightarrow \mathbb{R}$ be equal to $V_{T}[h]$ for all $T \in \mathcal{S}$ and $h \in \mathcal{E}_{T}$.

Theorem 5 The following two statements are equivalent:

1. $\succcurlyeq$ satisfies E1-E4, E5 $\bullet$

2. $\succcurlyeq$ has a utility representation $V: \mathcal{E} \rightarrow \mathbb{R}$ as described in Definition 7 , unique up to a positive affine transformation.

Proof [1 $\Longrightarrow 2$ 2] By the same argument as in the proof of Theorem 2, we can construct the utility representation $h \succcurlyeq z \Longleftrightarrow \bar{V}_{T}[h] \geq \bar{V}_{T}[z]$ for some functions $\bar{V}_{T}$, fixed up to a positive affine transformation. Let $\Delta\left(X^{*}\right)$ be as in the proof of Theorem 2 , then by E4.1, $\Delta\left(X^{*}\right)$ contains elements of the form $\left(x_{0}, x^{*}\right)$ for any $x_{0} \in X$ and by E5 $5^{\bullet}$, for all $x_{0} \in X, T \in \mathcal{S}$ and all $\left(x_{0}, \eta\right),\left(x_{0}, v\right) \in \Delta\left(X^{*}\right),\left(x_{0}, \eta\right)_{T} \sim\left(x_{0}, v\right)_{T}$.

Now, by the same argument, definitions and notation as in the proof of Theorem 2 we can rewrite $V_{T}\left(x_{0}, x\right)=g\left(x_{0}\right)+v_{T}\left(x_{0}, x\right)$. Condition (A.1) for functions $v_{T}$ is satisfied since the set $X^{*}$ contains all elements $\left(x_{0}, x^{*}\right)$ for which agent 0 is indifferent between the two groups.

[2 $\Longrightarrow$ 1] E1-E3 follow from the only if part of Theorem 8.4 of Fishburn (1970). E4.1 is a consequence of $v_{S_{1}}$ and $v_{S_{2}}$ being status functions. E4.2 is obvious. E5 directly follows from the property (A.1).

E6 and finiteness/compactness of $X$

It might look like that the requirements of E6 are not compatible with the set $X$ being finite or compact. Here is an example with $X=\{1,2,3\}$. Suppose, $(x, x)_{S_{1}} \sim(x, x)_{S_{2}}$ for all $x \in X$. Let $\succcurlyeq_{S_{1}}$ be defined, for example, by

$$
\begin{gathered}
(1,3) \sim(1,2) \sim(1,1) \\
\curlyvee \\
(2,3) \succ(2,2) \succ(2,1) \\
(3,3) \sim(3,2) \sim(3,1)
\end{gathered}
$$

For each $x \in X$ this satisfies E6.

\section{References}

Andreoni, J. (1995).Cooperation in public-goods experiments: Kindness or confusion? American Economic Review, 85(4), 891-904.

Anscombe, F., \& Aumann, R. (1963). A definition of subjective probability. Annals of Mathematical Statistics, 34(1), 199-205.

Auriol, E., \& Renault, R. (2008). Status and incentives. RAND Journal of Economics, 39(1), 305-326.

Ball, S., Eckel, C. C., Grossman, P. J., \& Zame, W. (2001). Status in Markets. Quarterly Journal of Economics, 116(1), 161-188.

Bell, D. E., \& Keeney, R. L. (2008). Altruistic utility functions for joint decisions. In S. Brams, W. V. Gehrlein, \& F. S. Roberts (Eds.), The mathematics of preference, choice and order: Essays in honor of Peter C Fishburn. New York: Springer. 
Bolton, G. E., \& Ockenfels, A. (2000). ERC: A theory of equity, reciprocity, and competition. American Economic Review, 90(1), 166-193.

Charles, K. K., Hurst, E., \& Roussanov, N. (2009). Conspicuous consumption and race. Quarterly Journal of Economics, 124(2), 425-467.

Costa-Gomes, M., \& Zauner, K. G. (2001). Ultimatum bargaining behavior in Israel, Japan, Slovenia, and the United States: A social utility analysis. Games and Economic Behavior, 34, 238-269.

Cummins, D. (2005). Dominance, Status, and Social Hierarchies. In D. M. Buss (Ed.), The handbook of evolutionary psychology, Chap. 20 (pp. 676-697). Hoboken: Wiley.

De Graaf, J., Wann, D., \& Naylor, T. H. (2005). Affluenza: The all-consuming epidemic. San Francisco: Berrett-Koehler Publishers.

Diestel, R. (2000). Graph theory (2nd ed.). New York: Springer.

Dufwenberg, M., Heidhues, P., Kirchsteiger, G., Riedel, F., \& Sobel, J. (2008). Other-regarding preferences in general equilibrium, mimeo, University of Arizona, University of Bonn, Université Libre de Bruxelles, Bielefeld University, UCSD.

Falk, A., Fehr, E., \& Fischbacher, U. (2008). Testing theories of fairness-Intentions matter. Games and Economic Behavior, 62, 287-303.

Fehr, E., \& Gächter, S. (2000). Cooperation and punishment in public goods experiments. American Economic Review, 90(4), 980-994.

Fehr, E., \& Schmidt, K. M. (1999). A theory of fairness, competition, and cooperation. Quarterly Journal of Economics, 114(3), 817-868.

Fishburn, P. C. (1970). Utility theory for decision making. New York: Wiley.

Frank, R. H. (1985). Choosing the right pond: Human behavior and the quest for status. New York: Oxford University Press.

Güth, W., Schmittberger, R., \& Schwarze, B. (1982). An experimental analysis of ultimatum bargaining. Journal of Economic Behavior and Organization, 3(4), 367-388.

Herstein, I., \& Milnor, J. (1953). An axiomatic approach to measurable utility. Econometrica, 21(2), 291297.

Kreps, D. M. (1988). Notes on the theory of choice, underground classics in economics. Colorado: Westview Press, Inc..

Layard, R. (1980). Human satisfaction and public policy. The Economic Journal, 90, 737-750.

Levine, D. K. (1998). Modeling altruism and spitefulness in experiments. Review of Economic Dynamics, 1(3), 593-622.

Luttmer, E. F. P. (2005). Neighbors as negatives: Relative earnings and well-being. Quarterly Journal of Economics, 120(3), 963-1002.

Maccheroni, F., Marinacci, M., \& Rustichini, A. (2008). Social decision theory: Choosing within and between groups, mimeo, Università Bocconi, Università di Torino, University of Minnesota.

Massey, D. S., Arango, J., Hugo, G., Kouaouci, A., Pellegrino, A., \& Taylor, J. E. (1993). Theories of international migration: A review and appraisal. Population and Development Review, 19(3), 431-466.

McCabe, K. A., Rigdon, M. L., \& Smith, V. L. (2003). Positive reciprocity and intentions in trust games. Journal of Economic Behavior and Organization, 52, 267-275.

Ok, E. A., \& Koçkesen, L. (2000). Negatively interdependent preferences. Social Choice and Welfare, 17, 533-558.

Postlewaite, A. (1998). Social status, norms and economic performances. The social basis of interdependent preferences. European Economic Review, 42, 779-800.

Rabin, M. (1993). Incorporating fairness into game theory and economics. American Economic Review, 83(5), 1281-1302.

Smith, A. (1759). The theory of moral sentiments. London: A. Millar.

Sobel, J. (2009). Generous actors, selfish actions: Markets with other-regarding preferences. International Review of Economics, 56, 3-16.

Starmer, C. (2000). Developments in non-expected utility theory: The hunt for a descriptive theory of choice under risk. Journal of Economic Literature, 38, 332-382.

Veblen, T. (1899). The theory of leisure class: An economic study of institutions. London: Allan and Unwin.

Vostroknutov, A., Tobler, P. N., \& Rustichini A. (2011). Causes of social reward differences encoded in human brain. Journal of Neurophysiology, forthcoming. 